\title{
The ionospheric signatures of flux transfer events and solar wind dynamic pressure changes
}

Article

Published Version

Lockwood, M., Cowley, S. W. H., Sandholt, P. E. and Lepping, R. P. (1990) The ionospheric signatures of flux transfer events and solar wind dynamic pressure changes. Journal of Geophysical Research, 95 (A10). p. 17113. ISSN 0148-0227 doi: https://doi.org/10.1029/JA095iA10p17113 Available at https://centaur.reading.ac.uk/38860/

It is advisable to refer to the publisher's version if you intend to cite from the work. See Guidance on citing.

Published version at: http://dx.doi.org/10.1029/JA095iA10p17113

To link to this article DOI: http://dx.doi.org/10.1029/JA095iA10p17113

Publisher: American Geophysical Union

All outputs in CentAUR are protected by Intellectual Property Rights law, including copyright law. Copyright and IPR is retained by the creators or other copyright holders. Terms and conditions for use of this material are defined in the End User Agreement.

www.reading.ac.uk/centaur 
Central Archive at the University of Reading

Reading's research outputs online 
THE IONOSPHERIC SIGNATURES OF FLUX TRANSFER EVENTS AND SOLAR WIND DYNAMIC PRESSURE CHANGES

\author{
M. Lockwood, ${ }^{1} 2$ S. W. H. Cowley, ${ }^{3}$ P. E. Sandholt, ${ }^{4}$ and R. P. Iepping ${ }^{5}$
}

Abstract. The generation of flow and current vortices in the dayside auroral ionosphere has been predicted for two processes ocurring at the dayside magnetopause. The first of these mechanisms is time-dependent magnetic reconnection, in "flux transfer events" (FTES); the second is the action of solar wind dynamic pressure changes. The ionospheric flow signature of an FTE should be a twin vortex, with the mean flow velocity in the central region of the pattern equal to the velocity of the pattern as a whole. On the other hand, a pulse of enhanced or reduced dynamic pressure is also expected to produce a twin vortex, but with the central plasma flow being generally different in speed from, and almost or thogonal to, the motion of the whole pattern. In this paper, we make use of this distinction to discuss recent observations of vortical flow patterns in the dayside auroral ionosphere in terms of one or other of the proposed mechanisms. We conclude that some of the observations reported are consistent only with the predicted signature of FTEs. We then evaluate the dimensions of the open flux tubes required to explain some recent simultaneous radar and auroral observations and infer that they are typically 300 $\mathrm{km}$ in north-south extent but up to $2000 \mathrm{~km}$ in longitudinal extent (i.e., roughly 5 hours of MUT). Hence these observations suggest that recent theories of FIFs which invoke time-varying reconnection at an elongated neutral line may be correct. We also present some simul taneous observations of the interplanetary magnetic field (IMF) and solar wind dynamic pressure (observed using the IMP8 satellite) and the ionospheric flow (observed using the EISCAT radar) which are also only consistent with the FTE model. We estimate that for continuously southward $\operatorname{IMF}\left(\left\langle\mathrm{B}_{\mathrm{p}}\right\rangle \approx 5 \mathrm{nT}\right)$ these FTEs contribute about $30 \mathrm{kV}$ to the mean total transpolar vol tage $(\sim 30 \%)$.

\section{Introduction}

Much recent interest has been focused on the coupling of the dayside auroral ionosphere to the

\footnotetext{
${ }^{1}$ Rutherford Appleton Iaboratory, Chilton, Didcot, England.

${ }^{2}$ Also at Blackett Laboratory, Imperial College, Iondon.

${ }^{3}$ Blackett Iaboratory, Imperial College, Iondon.

${ }^{4}$ Department of Physics, University of Oslo, Oslo, Norway.

${ }^{5}$ Laboratory for Extraterrestrial Physics, Goddard Space Flight Center, Greenbelt, Maryland.
}

Copyright 1990 by the American Geophysical Union.

Paper number 90JA01212. 0148-0227/90/90JA-01212\$05.00 magnetopause (see review by Lockwood and Cowley [1988]). In particular, the ionospheric signatures of two transient magnetopause processes have been predicted, namely time-dependent magnetic reconnection ("flux transfer events") and magnetopause motions induced by dynamic pressure changes in the magnetosheath.

Characteristic signatures in the magnetic field and particle populations near the magnetopause have been interpreted in terms of flux transfer events (FTEs) for over a decade now [Russell and Elphic, 1979; Paschmann et al., 1982; Berchem and Russel1, 1984; Rijnbeek et al., 1984; Saunders et al., 1984; Southwood et al., 1986; Farrugia et al., 1988]. This interpretation calls for the dayside auroral ionosphere to exhibit flow and current signatures [Southwood, 1985, 1987; Cowley, 1984a, 1986; Lee, 1986]: in fact, were such. signatures proven to be absent, this would cast doubt upon the accepted model of the magnetopause FWE signatures invoking magnetic reconnection. In addition, if detected and understood, ionospheric signatures of FWEs offer a unique opportunity to quantify the full voltage which they apply to the coupled magnetosphere-ionosphere system. Two types of signature have been predicted, based on different interpretations of the magnetopause observations, but both invoking magnetic reconnection. The Southwood [1985, 1987] predictions are based on the patchy, sporadic reconnection model of Russell and Elphic [1978, 1979], but are readily generalized to include the concept of time-dependent reconnection at a single, elongated reconnection neutral line, as advanced by Southwood et al. [1988] and Scholer [1988]. These models require a pair of oppositely directed field-aligned currents flowing on the flanks of the region of newly opened flux, giving a twin vortical flow pattern in the ionosphere, as illustrated by the equipotentials in Figure 1a. Lockwood and Freeman [1989] have pointed out that the ionospheric footprints of newly opened flux tuibes will tend to move initially east or west (depending on the interplanetary magnetic field (IMF) $\mathrm{B}_{\mathrm{y}}$ component) under the action of magnetic tension, ${ }^{\mathrm{b}}$ before their motion evolves toward poleward, under the influence of antisolar magnetosheath flow. This concept was also used by Saunders [1989] in his explanation of the cusp field-aligned currents. Hence the orientation of the open/closed field line boundary will evolve, relative to the event velocity, $v_{e}$, between the dashed lines shown in Figure 1a. The Southwood model of the twin vortex ionospheric flow signature of an FWE is based on the concept that a newly opened flux tube moves faster than the surrounding flux tubes (be they closed or "older" open flux tubes). If the newly opened flux tube only moved at the speed of the background flow, there would be no vortical flow features. Southwood predicts that shortly after reconnection, a newly opened FTE flux tube in the ionosphere will move faster than surrounding flux 


\title{
NORTHERN HEMISPHERE FLOW PATTERNS
}

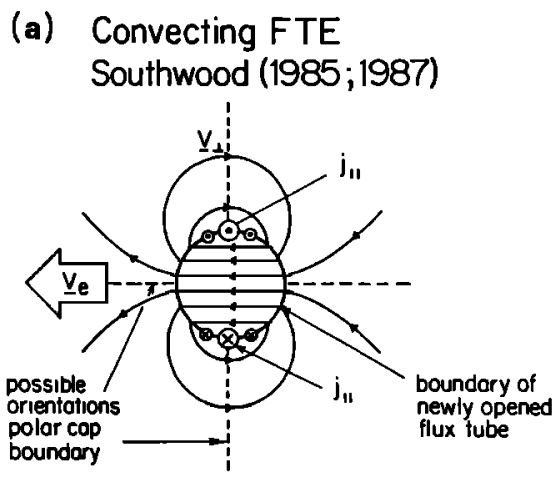

(c) General FTE

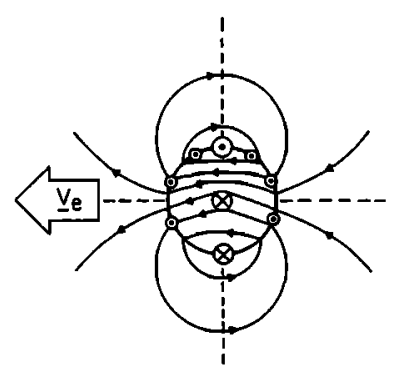

(b) Rotating FTE

Lee (1986)

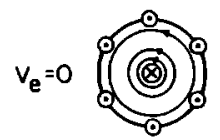

\begin{abstract}
Fig. 1. Snapshots of flow equipotentials for various predicted signatures of magnetopause processes in the northern hemisphere ionosphere. The vectors in and out of the plane of the diagram are field-aligned currents, and the dashed lines are possible orientations of the polar cap boundary. The circular region in the FTE models is the region of newly opened magnetic flux. The pattern as a whole moves with velocity ve, whereas plasma flow at a given point is $v_{\perp}$.
\end{abstract}

tubes, thereby pushing "older" open flux out of the way and overtaking them (as discussed by Lockwood et al. [1988b]) and giving the twin vortical flow pattern. However, after the FiE tube has unbent, Southwood points out that it is like any other lobe tube (i.e., it has become one of the "older" open flux tubes) and can no longer overtake surrounding tubes: in other words it no longer excites a twin vortex. Lockwood et al. [1990] have shown that the ionospheric response times to changes in the IMF, as measured by the EISCAT-AMPTE observations and analyses of geomagnetic disturbance, require that newly opened flux tubes only excite plasma flows for a limited period $(\sim 10 \mathrm{~min})$; consequently this is roughly the expected lifetime of any Southwood FTE twin vortex before it is subsumed into the polar cap flow [Lockwood and Cowley, 1988]. Likewise, precipitation of magnetosheath particles is only found on newly opened flux tubes (the cusp) and not on older open flux tubes (the remainder of the polar cap). This is usually attributed to the fact that ions can only be injected across the magnetopause where the magnetosheath flow at the boundary is sub-Alfvénic, i.e., on open field lines which are close to the subsolar stagnation point [Reiff et al., 1977]. Hence we would expect strong, twin vortical flows and enhanced $630-\mathrm{nm}$ aurora to be established shortly after an FIE was reconnected and both to decay to values typical of the polar cap within a short lifetime (of order 10 min).

A second type of FIE signature has been predicted by Tee [1986], based on generation of FTEs by multiple, elongated reconnection neutral lines on the magnetopause. This produces a tight twist in the magnetic field near the magnetopause, generating a coaxial field-aligned current system [Saunders et al., 1984] and a single flow vortex in the ionosphere, as shown in Figure $1 \mathrm{~b}$. Wright [1987] argued topologically that the initial shear in the field will impose a half twist on a reconnected flux tube which will subsequently untwist. Southwood et al. [1988] have shown that such a twist will also arise from a velocity shear. Hence a general FTE signature will inevitably have features of both flux tube convection and rotation: the imposed twist will unwind in the Southwood model, and translational motion of a Lee flow vortex would require the field-aligned currents of the Southwood model. Hence convective and rotational signatures will be superposed [McHenry and Clauer, 1987; Bering et al., 1988]. However, a very important feature of a general FTE signature in the ionosphere (an example of which is given in Figure 1c) is that untwisting gives no flow at the center of the event and hence the flow there must be that given 
by the convecting FTE model, i.e., that of the event as a whole. The same statement can be made about the mean of the velocity, averaged over the entire newly opened flux tube. It should also be noted that background convection in the same/opposite direction to $\mathrm{v}$ will cause the flow outside the newly opened flux tube to be weakened/strengthened, respectively.

Recently, a number of authors have noted signatures of vortical flow in the iunosphere which appear consistent with neither of the above FTE models, nor with a general combination of them (see the following section). Comparison with solar wind data has indicated that such effects may sometimes, but not always, have been generated by solar wind dynamic pressure changes, and some events occurred during periods of nor thward IMF, i.e., when FIEs are not expected and rarely observed. This has generated much debate about the role of dynamic pressure pulses either in the solar wind [Sibeck et al., 1989b, c; Ianzerotti, 1989; Bering et al., 1990] or in the magnetosheath when the IMF is near radial [Fairfield et al., 1990]. Elphic [1988] pointed out that the ionospheric signatures were qualitatively consistent with the expected magnetopause boundary motions in response to dynamic pressure changes, and Glassmeier et al. [1989] sketched the fieldaligned currents and flows required to explain their ionospheric observations. These two elements have been unified into a consistent theory by Southwood and Kivelson [1990] and L.C. Iee (Generation of field-aligned currents by pressure pulses in the magnetosheath, submitted to Geophysical Research Letters, 1990) (hereinafter referred to as Lee, submitted, 1990), who have shown how the boundary motions can give vortical flows in the ionosphere, only if there are spatial gradients near the equatorial magnetopause, which cause the pressure pulse to generate field-aligned currents into/out of the ionosphere. Without such gradients, the pressure pulse would cause a compression of the equatorial magnetopause, but because the compression is negligible in the ionosphere, the compressional wave would be almost perfectly reflected by the ionosphere and the ionospheric flows would be virtually zero. As a result, there is no simple relationship between the speed of motion of the equatorial magnetopause and the ionospheric flow speed. To produce a twin vortex (and hence in some respects mimic a convecting FTE signature) there must be a pulse of enhanced or decreased solar wind pressure. Figure $1 d$ is a sketch of the resulting field-aligned currents and flow pattern from the model computations of Iee (submitted, 1990). This shows that the flow at the center of the twin vortex is orthogonal to the background polar cap boundary, along which the event propagates. In general, we expect the phase velocity of the twin vortex pattern, $v_{e}$, will be at a large angle to the plasma flow at the event center. Furthermore, for the typical input parameters employed by Lee, along with height-integrated Hall and Pedersen conductivities of $\Sigma_{H}=10$ mhos and $\Sigma_{P}=5$ mhos, respectively, both the theory and the magnetometer observations of Friis-Christensen et al. [1988] yield a flow speed of $300 \mathrm{~m} \mathrm{~s}^{-1}$ at the center of the event (for a $2 \mathrm{nPa}$ pulse in dynamic pressure): by contrast, $v_{\text {is }}$ is observed and predicted to be $6 \mathrm{~km} \mathrm{~s}^{-1}$. For lower conductivities the flow speed will be correspondingly higher.
Hence, in general, the flow at the event center for a dynamic pressure pulse twin vortex is different from the motion of the whole pattern, both in direction and speed. These factors provide an important distinction between the twin vortical flow signatures in the ionosphere predicted for FTEs and dynamic pressure pulses.

It is interesting to note that, as originally suggested by Dessler [1964], both increases and decreases in dynamic pressure cause anti-sunward transfer of closed field lines, but with no transfer of matter across the magnetopause [Southwood and Kivelson, 1990; D. J. Southwood, private communication, 1989]. The temporal variability of "viscous like" flows at the ionospheric convection boundary, inferred from continuous ground-based radar observations by Lockwood et al. [1988a], suggests that the mechanism responsible may indeed occur in bursts, as would be predicted for pulses in dynamic pressure. The fact that solar wind dynamic pressure variability is the same for northward and southward IMF [Bowe et al., 1990], limits the total vol tage which this mechanism can contribute to the total trans-polar potential difference to below the peak value of about $30 \mathrm{kV}$ observed during northward IMF (see reviews by Cowley [1984b] and Reiff and Luhmann [1986]). This statement must be valid provided there is no difference in magnetospheric morphology for northward and southward directed IMF which alters the terrestrial response to solar wind dynamic pressure variations (D. Sibeck, private communication, 1989). One such difference could possibly be the inferred variation in the thickness of the low-latitude boundary layer with IMF $B_{3}$ (see review by Lundin [1988]).

The ${ }^{\mathrm{Z}}$ contribution to the total transpolar voltage by FTEs is not known. Interpretation of magnetopause data using the Russell and Elphic [1978, 1979] circular flux tube model yields estimates of typically $10-20 \mathrm{kV}$ for the reconnection voltage associated with each event (i.e., along the $X$ line). However, the models by Lee and Fu [1985], Southwood et al. [1988] and Scholer [1988] multiply this estimate by a factor roughly equal to the ratio of the length of the elongated neutral line to the diameter of the Russell and Elphic [1978;1979] flux tube. Saunders et al. [1984] found a typical event diameter of about $1 R_{F}$ in the boundary normal direction. If the $\mathrm{X}$ line $\mathrm{E}_{\text {is }}$ in fact $5-10 \mathrm{R}_{\mathrm{F}}$ in length, the new FTE theories therefore will predict reconnection voltages of order $50-100 \mathrm{kV}$. However, it should be noted that reconnection at these voltages is estimated to last for typically $2 \mathrm{~min}$, whereas FIEs recur only every $8 \mathrm{~min}$ on average. Hence the contribution to the time-averaged transpolar voltage would generally be only a quarter of the reconnection voltages quoted above.

In the next section we briefly review direct and indirect observations of vortical flow patters in the ionosphere. In the light of the above discussion, it can be seen that the correct interpretation of these observations is vital if the total voltage contributed by the two proposed magnetopause mechanisms is to be quantified. Then in the third section, we discuss the dimensions of the newly opened flux tube required to explain some of the recently published observations in terms of FTEs. The results reveal a surprising elongation along the polar cap boundary, which is 
shown in the fourth section to give the event the appearance of a flow channel (and current electrojet) with only weakly vortical features. We then present an event which is shown to be consistent with a near-circular FWE model. Iastly we discuss these observations in terms of FTE and dymamic pressure pulse theories.

\section{Previous Observations of Flow Vortices in the Dayside Auroral Ionosphere}

The first ionospheric observations which were associated with one of the two processes discussed above were by van Eyken et al. [1984] and Geortz et al. [1985]. The former suggested that a 10-min burst of poleward flow observed by the European Incoherent Scatter (EISCAT) radar was an FTE. In the latter, data from the Scandinavian Twin Auroral Radar Experiment (STARE) were described in terms of bursts of flow into the polar cap across the convection boundary reversal and were interpreted in terms of an FTE model, because theparticle dropouts, seen simultaneously by the conjugate, GEOS satellite, were not consistent with a compressional motion of the magnetopause over the satellite. Further radar and magnetometer observations of vortical flow signatures by Todd et al. [1986] and Lanzerotti et al. [1987] were shown to be consistent with FIE effects, but subsequently Sibeck et al. [1989a] have shown that these could be due to dynamic pressure changes which were also observed in the upstream solar wind (see also Ianzerotti [1989] and Sibeck et al. [1989b]).

Signatures seemingly consistent with the dynamic pressure pulse model (Figure 1d) have been found. Friis-Christensen et al. [1988], using data from the Greenland magnetometer chain, deduced that a large twin vortex had propagated westward (antisunward) around the morning sector polar cap boundary at about $5 \mathrm{~km} \mathrm{~s}^{-1}$. These authors noted that this event was inconsistent with an FIE because the flow at the event center was nearer northward than westward. The peak deflection of the magnetic field was about $200 \mathrm{nT}$, equivalent to a flow speed in the center of the event of about $300 \mathrm{~m} \mathrm{~s}^{-1}$ for a Hall conductivity $\Sigma_{\mathrm{H}}$ of 10 mhos. This speed estimate would increase in proportion with lower estimates of $\Sigma_{H}$. Variations in both solar wind dynamic pressure and the IMF $B$ component were observed shortly before this $\mathrm{y}^{\mathrm{y}}$ event. The dynamic pressure was observed to rise by $50 \%$ suddenly and then equally rapidly to fall by $40 \%$ after about $150 \mathrm{~s}$. Iee (submitted, 1990) has pointed out that this is consistent with the nature and duration of the event observed in the ionosphere. Similar events have been reported by Glassmeier et al. [1989], using data from

Scandinavian magnetometers. These have typical phase speeds, $v_{e}$, of about $2.5 \mathrm{~km} \mathrm{~s}^{-1}$, but much lower inferred poleward flow speeds $\left(\approx 60 \mathrm{~m} \mathrm{~s}^{-1}\right)$ : however, the latter may be lower than in the case discussed by Friis-Christensen et al. because the observations only covered the equatorward portion of the events. Single vortices could occur in isolation for a step function change in dynamic pressure (although the flows associated with the necessary opposite and equal field-aligned current must be present at some location) or can be one of a long string of events for oscillations in dynamic pressure, as has been observed by McHenry et al. [1988]. Multiposition observations have indicated that these types of ground magnetic perturbation can indeed be caused by dynamic pressure changes [Potemra et al., 1989; Farrugia et al., 1989]. Farrugia et al. [1989] directly observed the magnetopause contraction caused by the observed increase in solar wind dynamic pressure as well as observing the impulsive response at a wide variety of ground-based magnetometer stations on the dayside at high latitudes. However, not all impulsive auroral events seem to be attributable to pressure pulses: Bering et al. [1990] present two cases where IMP 8 failed to detect any pressure pulse and there are other examples (E. Friis-Christensen, private communication, 1989). However, in such cases the region of enhanced pressure could have been small enough to have impacted on the bow shock and magnetopause, without passing over IMP 8. A problem with associating observed pressure pulses with ionospheric events is that there will always be some fluctuation level in solar wind dynamic pressure and we-must therefore invoke theory to define what is a significant pulse or level of "buffeting" and must ensure that there are no larger pressure fluctuations which fail to generate an ionospheric event. If pressure pulses of some magnitude are always present, it will always be possible to associate any ionospheric event with a pulse, even when there is no causal relationship.

Recent observations by the EISCAT radar and surrounding magnetometers have revealed flow bursts in the ionosphere which do not appear to belong to the above class of traveling disturbance [Lockwood et al., 1989a; Sandholt et al., 1990]. These events are observed during transient dayside auroral displays, which had previously been associated with FTEs (see review by Sandholt [1988]). Comparison with data from a chain of magnetometers suggests that this flow burst is at the center of a twin vortical flow perturbation pattern. In these cases, the flows observed by EISCAT are at all times very similar to the motion of the events as a whole (as given by the radar, magnetometer and optical observations).

Furthermore, these ionospheric plasma flows are large (typically 2-3 $\mathrm{km} \mathrm{s}^{-1}$ westward initially, evolving to around $1 \mathrm{~km} \mathrm{~s}^{-1}$ poleward). These events are therefore well described by the Southwood FIE model considering the evolution of newly opened flux tubes predicted by Lockwood and Freeman [1989] and Saunders [1989]: but they do not appear to be consistent with the pattern for dynamic pressure changes. From the limited data available, Lockwood et al. [1989b] have shown that their occurrence as a function of the IMF $B_{c}$ is as expected for FIEs in that they occur every ${ }^{2} 8$ min when the IMF is consistently southward or following isolated swings to southward IMF. The total voltage in these events is large. The EISCAT/optical observations show that the arcs move with the local ionospheric electric field, and hence, for example, the arc discussed by Sandholt [1988], which was moving poleward at $1 \mathrm{~km}$ $\mathrm{s}^{-1}$ and was $1000 \mathrm{~km}$ long in east-west extent, is associated with a voltage of at least $50 \mathrm{kV}$. The minimum estimate of the total potential difference across the westward flow channel of the largest event observed by Lockwood et al. [1989a] is 80 $\mathrm{kV}$. In the light of these EISCAT observations, 
Lockwood and Smith [1989, 1990] have been able to interpret a cusp particle injection event, seen by the DE satellites, in terms of an FIE. In the following section, we take a close look at the data from the EISCAT radar during the events reported by Lockwood et al. [1989a, b] and Sandholt et al. [1990] in an attempt to define the spatial dimensions of the open flux tubes required for the FIE model.

\section{Transient Dayside Auroral/Flow Burst Events and Their Spatial Extent}

Figures $2 \mathrm{a}$ and $2 \mathrm{~b}$ are reproduced from Lockwood et al. [1989a] and show the data used by these authors to define the transient auroral and flow burst events. These data were obtained on January 12, 1988, by the EISCAT radar (operating in Cormon Programme CP-4 mode which is virtually identical to the "Polar" experiment mode described by van Eyken et al. [1984] and Willis et al. [1986]) and by the meridian-scanning photometers at $\mathrm{Ny}$ Alesund, Spitzbergen [Sandholt et al., 1985, 1989a, b, 1990]. In the Polar mode, EISCAT operates monostatically from the Troms $\emptyset$ site and swings the beam at low elevation $\left(21.5^{\circ}\right)$ between two azimuths $12^{\circ}$ either side of the northward normal to the I shell. The signal is divided into range gates $75 \mathrm{~km}$ in length, and line-of-sight

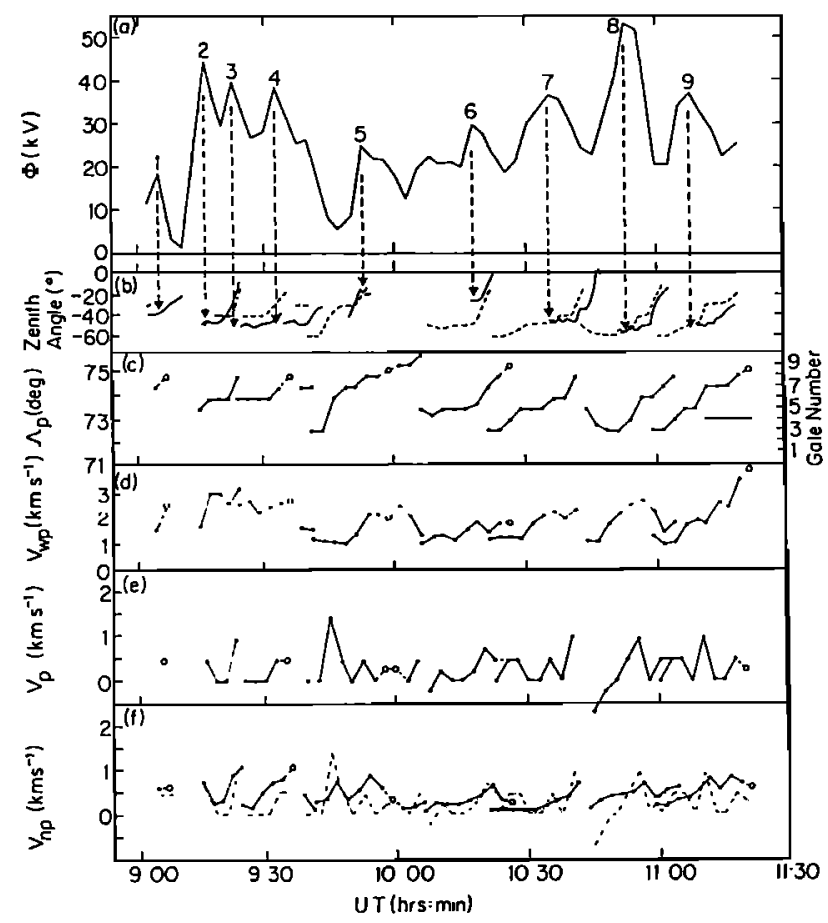

Fig. 2. Transient aurorae and flow bursts observed by a 630-nm meridian-scanning photometer at $\mathrm{Ny}$ Alesund, Spitsbergen, and by the EISCAT radar on January 12, 1988. (a) The potential, $\Phi$, across gates 1-7 of the EISCAT field of view. (b) The zenith angle (positive north) of peak $630-\mathrm{nm}$ emissions exceeding $3 \mathrm{kR}$. (c) The latitude, $\Lambda_{p}$, of peak westward flow observed by EISCAT. (d) and (f) The westward and northward plasma velocities at $\Lambda=\Lambda_{p}, V_{\text {wp }}$ and $V_{n p}$, respectively. (e) The northward speed of the peak of westward flow, $V_{p}$, which is also shown as a dot-dash line in Figure $2 f$. velocities for the two beam directions are then combined to give a flow vector for each gate. The vectors are ascribed to points midway between the two beam directions which form a meridional chain, roughly $150 \mathrm{~km}$ to the west of the meridian scanned by the photometers. Maps of the locations of the centers of the radar gates and of the optical observations at a given zenith angle have been given by Lockwood et al. [1989a, b] and Sandholt et al. [1990]. The beam-swinging cycle time is 5 min, and field-perpendicular flow vectors for each range gate are derived every $2.5 \mathrm{~min}$ using the procedure described by Willis et al. [1986]. This procedure makes use of postintegrated data for each 2-min dwell of the antenna at each azimuth. Analysis of errors in line-of-sight velocity determination reveals that if the signal/background ratio exceeds $0.5 \%$, errors are low $\left(\sim \pm 25 \mathrm{~m} \mathrm{~s}^{-1}\right)$ (the experiment succeeds on such low signal levels because it is run similtaneously on five different frequency channels) and for all data presented this condition is met. Note that some line-of-sight velocities for 10-s integration periods are also presented: these only have the required signal/background ratio for the nearer range gates, and good data are not available as far to the north as for the 2-min integrations used in the derivation of the vectors. Figure $2 a$ shows the potential across the north-south dimension of the EISCAT field of view, $\Phi$, as a function of UT. This potential is derived by converting the observed westward flow speed into a northward electric field, with the assumption that the ion gas is drifting with a velocity $\mathrm{v}=\mathrm{E} \times \mathrm{BB} / \mathrm{B}^{2}$. The design of the Polar experiment means that observations are made at altitudes above $211 \mathrm{~km}$, where this assumption is valid because wind effects are negligible. The northward component of the electric field is then integrated across the radar field of view (taken here to be the first seven range gates, for which sufficient signal strengths were received throughout the period). (Note that for the first two data points, signals of insufficient signal/background ratio were received in gate 1, and the value of the westward flow speed was obtained by extrapolation (see Figure 3): the uncertainty introduced into these two values of $\Phi$ is estimated to be only about $1 \mathrm{kV}$ as flow speeds were low in the nearer range gates.) It should also be noted that there is an important limitation of these flow and potential data in that they are derived using the beam-swinging technique. This effect will introduce errors into the derived flows in the presence of large spatial and temporal gradients in the real flow (see Lockwood et al. [1988a] and Etemadi et al. [1989], respectively). Etemadi et al. have shown that in the presence of dominant eastward or westward flow (as in the data discussed here) the main effect of rapid variations is to introduce smoothing into the sequence of flow speeds derived by Polar. However, if there is also significant north-south flow, changes in one flow component will generate spurious derived flows in the other component.

Figure 2b shows the zenith angle at which peak 630-nm emission was simul taneously observed by photometers at Ny Alesund, Spitzbergen, which scan the magnetic meridian from horizon to horizon every $18 \mathrm{~s}$ (negative values corresponding to locations to the south of Ny Alesund). Only peak 
intensities exceeding $3 \mathrm{kR}$ are shown so that the transient arcs can be distinguished from the lower intensity, persistent background cusp/cleft aurora. It can be seen from the dashed arrows that each peak in potential occurred close to the onset of an intense, transient 630-nm aurora at the photometer meridian. Sandholt et al. [1990] have shown that initially the second transient arc moved across the meridian of the photometer observations from the east, before slowing and moving poleward. As discussed by Lockwood [1989b], the first event was the only one not to behave this way, in that the initial westward flow was much weaker than for all the other events: the westward plasma flow seen by the radar was correspondingly weaker for this one event. The peak $\Phi$ is observed by the radar during the initial, westward motion, phase when the peak $630-\mathrm{nm}$ emission remains at roughly constant zenith angle, but is increasing in intensity. The poleward moving phase of each event can be seen in Figure $2 b$ as the peak intensity moves toward zero zenith angle.

Sandholt et al. [1990] have studied the first and second event in detail and state that the northward and westward flow components observed by EISCAT in the range gates nearest to the auroral luminosity were at all times very similar to those of the transient aurora. In view of the significance of this result, we here elaborate on the basis of Sandholt et al.'s statement. The larger event described by Sandholt et al. Was event 2, for which they tracked the leading edge of the auroral transient between successive 1-s images taken by the all-sky TV camera. This was possible for 0920:55 and 0922:00 UT. Remembering that the all-sky TV camera is predominantly sensitive to 557.7-nm emissions, the location of this edge was mapped assuming an emission al titude of $130 \mathrm{~km}$. Sandholt et al. found it had moved west, parallel to the $\mathrm{L}$ shells by $201 \mathrm{~km}$ : this corresponds to a mean speed of $3.1 \mathrm{~km} \mathrm{~s}^{-1}$ in this period. The ion flows derived from the radar data closest in time to these images are for 0921:30. At this time a westward flow component of $3.00 \mathrm{~km}$ $\mathrm{s}^{-1}$ was observed for the furthest usable range gate (gate 7) which was only about $0.25^{\circ}$ of invariant latitude south of the transient $557.7-\mathrm{nm}$ arc (and within the 630-nm arc). To derive the northward speeds, Sandholt et al. used the peak intensity of the $630-\mathrm{nm}$ transient because this was observed by the photometer for longer than the 557.7-nm transient and hence northward speed could be calculated more accurately. Between 0921:35 and 0924:10 the mean poleward speed was $0.7 \mathrm{~km} \mathrm{~s}$ which can be compared with the radar observations of the northward plasma flow of $0.617 \mathrm{~km} \mathrm{~s}^{-1}$ at $0921: 30$ and $0.942 \mathrm{~km} \mathrm{~s}^{-1}$ at 0924:00 (for gate 7). Taking the mean of these values we obtain $0.78 \mathrm{~km}$ $\mathrm{s}^{-1}$. The other event (event 1 ) studied by Sandholt et al. did not show clear westward motion, and so this component could not be quantified from the all-sky TV camera. The camera and photometer yielded a northward speed of $1.2 \mathrm{~km} \mathrm{~s}^{-1}$ for the period 0910:00-0912:00 and exactly the same value was obtained from the 630-nm aurora for the period 0909:33-0911:43. The closest radar data point is 0911:30 for gate 7, and the northward flow speed derived was $1.21 \mathrm{~km} \mathrm{~s}^{-1}$. Again this gate was within the 630-nm transient and is just equatorward of the 557.7-nm transient.
Consequently, Sandholt et al. conclude the arc motion and the local plasma motion are the same. This comparison is subject to some experimental uncertainties, principally because of the assumption of emission altitudes and the radar beam-swinging technique. However, the similarities of northward speeds derived from the photometer data indicate that the former is not a major source of error, and the ion temperature rises observed by EISCAT show that the flow magnitudes derived from the beam-swinging technique are largely correct.

The dashed lines in Figure $2 \mathrm{~b}$ and the remainder of Figure 2 will be discussed later.

Magnetometer data from Ny-Ålesund, Hornsund, Børnøya and Tromsø were also recorded during this period [see Sandholt et al., 1990]. Hornsund and Børnøya lie within the Polar field of view and showed deflections consistent with the northward electric field (westward flow) observed by the radar. However, $\mathrm{Ny}-\AA$ llesund (to the north of the radar field of view) and Troms 0 (to the south) both observed southward field deflections (corresponding to swings toward eastward flow). Hence during each event, the magnetometers show a perturbation with a double reversal with eastward flow to the north and south of the westward flow burst. As pointed out by Lockwood et al. [1989a] and Sandholt et al. [1990], the double reversal is a feature of a westward moving Southwood model FTE twin vortex. However, the magnetometer signatures are not as predicted for the Southwood FTE model by McHenry and Clauer [1987]. Lockwood et al. [1989a] list a number of reasons why the magnetometer observations may differ from these predictions. Firstly, McHenry and Clauer only considered one event in isolation. In practice, a magnetometer integrates over a region of radius about $300 \mathrm{~km}$. Hence when events recur rapidly, there can be more than one event influencing the magnetogram at any one time. We believe this effect causes Troms $\varnothing$ to observe a smooth bay between 0900 and 0945 when the events recur every 8 min. After 0945, Troms $\emptyset$ resolved individual events because they are $20 \mathrm{~min}$ apart on average. This behavior should be compared with that seen at Hornsund which was very much closer to the transient 557.7-nm aurora and where distinct impulsive events were resolved for all events. The second major complicating factor is that McHenry and Clauer assumed the ionospheric conductivities to be uniform. This is clearly invalid in the presence of the auroral transients during these events (see Sandholt et al. [1990] for estimates of the conductivity enhancements). Furthermore, this spatial structure is highly time dependent and moves with the event. The importance of conductivity changes was demonstrated by Lockwood et al. [1989b]. They showed that the impulsive magnetometer signatures at Hornsund occurred on average 7 min after the peak electric field observed by the radar and this corresponded to the average delay before the 557.7-nm arc reached the longitude of Hornsund as it moved westward. As a result, these authors concluded that the magnetometer signatures were strongly influenced by the conductivity enhancements associated with the 557.7-nm arc. Thirdly, in the predictions the events were assumed to move with uniform velocity over the entire magnetometer field of view; this is not the case for the events observed (auroral 
and plasma flow) whose velocity continuously evolves from about $3 \mathrm{~km} \mathrm{~s}^{-1}$ westward to $1 \mathrm{~km} \mathrm{~s}^{-1}$ poleward. Iastly, we note that the predictions were for a circular FIE flux tube, which is an assumption we will question later in this paper. In view of these complicating factors it may be impossible to unambiguously define an FTE twin vortex from magnetometer data (rather they may well show transient electrojet like features). We believe high time resolution radar observations over a wide field of view will be required.

Lockwood et al. [1989b] have studied the IMF $B_{3}$ component during these events, as observed by the $\mathrm{Z}$ IMP 8 satellite. When the IMF was consistently southward (0900-0945 UT), the events were found to recur every $8.3 \pm 0.6 \mathrm{~min}$, very similar to the repetition rate of FTEs at the magnetopause under the same IMF conditions [Berchem and Russell, 1984; Rijnbeek et al., 1984]. After 0945 UT, the IMF was predominantly nor thward and made only isolated excursions to a southward orientation, each lasting several minutes. By making allowance for the propagation time between IMP 8 and the radar, Lockwood et al. were able to associate each transient auroral/flow burst event after 0945 UT with a southward swing of the IMF. The IMF $B_{4}$ component was strongly positive for this period $y$ $(\approx 10 \mathrm{nT})$, and hence the westward and then northward flow is as predicted for newly opened flux tubes by Lockwood and Freeman [1989] and Saunders [1989]. We conclude that the occurrence and motion of these events are well explained in terms of transient magnetic reconnection.

Iockwood et al. [1989a] used a flow model of the kind shown in Figure la to infer the dimensions of the event with the largest voltage (labeled 8 in Figure 2). The north-south dimension was also found to be largest for this event, being estimated to be as great as $800 \mathrm{~km}$ using data from the radar, photometers and magnetometers. The east-west dimension, on the other hand, was derived with the assumption that the center of a flow pattern of the kind shown in Figure la passed close to the radar field of view. In this case westward flows would be seen to rise before the open flux tube intersected the radar field of view, be constant while the radar was within the open flux tube and then fall as the event moved westward of the field of view. This behavior is observed in the total potential, $\Phi$, in Figure 2. Event 8 , for example, gives peak $\Phi$ lasting $2_{i} 5$ min: for the observed flow speeds of $2 \mathrm{~km} \mathrm{~s}^{-1}$, Lockwood et al. estimated the open flux tube to have been $300 \mathrm{~km}$ in east-west extent. This small east-west dimension, however, is not consistent with the auroral TV images. For example, Sandholt et al. [1990] show that event 2 is associated with a green line $(557.7-\mathrm{nm})$ arc which is over $500 \mathrm{~km}$ in east-west extent, whereas the above procedure predicts the event is at most $400 \mathrm{~km}$ wide. The argument given by Lockwood et al., however, does not take account of a number of factors. Firstly, the analysis of Etemadi et al. [1989] shows that there will be considerable smoothing of the sequence of $\Phi$ and hence the radar will have been within the open flux tube for longer than the period of peak $\Phi$. Secondly, analysis of the flows caused by elliptical regions of newly opened flux shows that a much greater fraction of the westward flow peak may be within the open flux tube than for the circular tube case (see following section). Iastly, structure in the flow within the radar field of view was not considered, and this may influence estimates of the north-south extent of the events.

Figure 3 shows latitude profiles of westward flow speed $V_{w}$ as a function of UT, for the period covered by Wigure 2. The parameter $\Phi$ is an integral of these flows between gates 1 and 7 , and it can be seen that there is indeed structure within the radar field of view which cannot be determined from $\Phi$. Peak westward flows which exceed $1 \mathrm{~km} \mathrm{~s} \mathrm{~s}^{-1}$ have been joined by dashed lines to guide the eye. The dashed lines become dotted when the peak westward flow reaches the furthest range gate observed. If $\mathrm{V}$ for a gate adjacent to a peak is within $100 \mathrm{~m} \mathrm{~s}^{-1 \mathrm{w}}$ of the peak value, the dashed line is drawn midway between these two gates. We acknowledge that there are places in the sequence where the dashed lines could follow more than one path. However, there are also times when only one peak in westward flow was observed and this peak clearly migrated poleward. If we

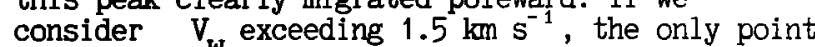
at which the ${ }^{w}$ path of a dashed line is ambiguous is at 0925 UT: here the optical data (Figure 2b) support our interpretation of a third flow channel forming while the second one moves poleward. The data are certainly consistent with a series of enhanced westward flow "channels" moving poleward. It is difficult to resolve at exactly what time a flow channel formed, but in all cases it moved poleward out of the field of view rather than decaying within it. Note that event 6 is at a sufficiently low latitude early in its lifetime
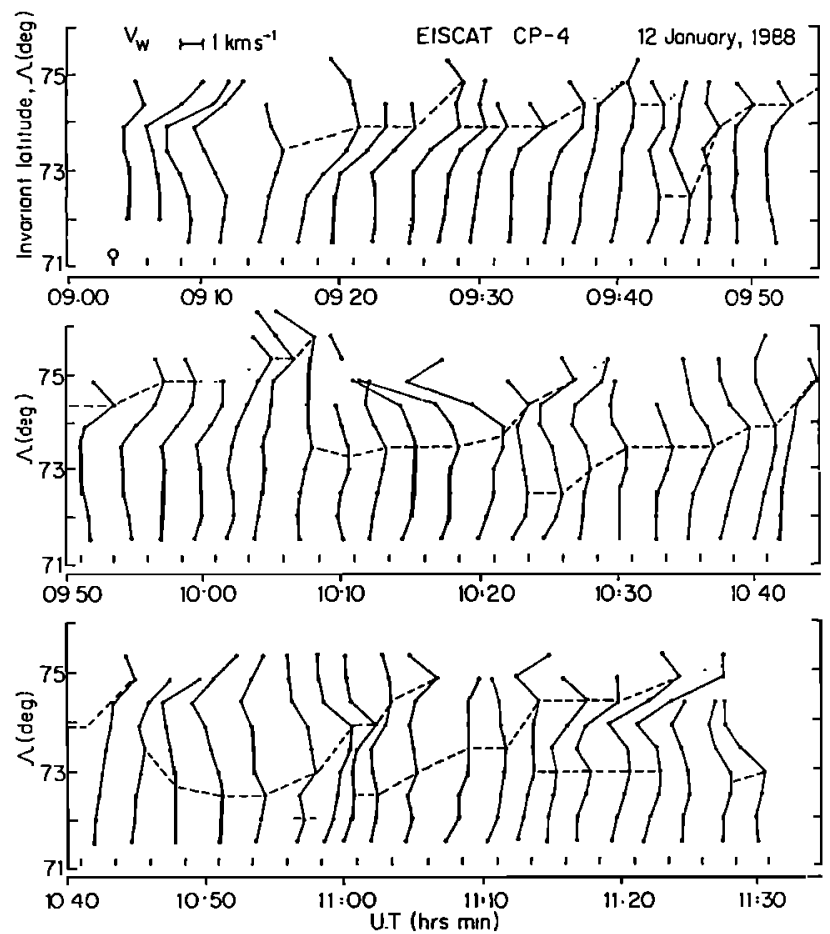

Fig. 3. Invariant latitude profiles of westward flow velocity, $V_{W}$, as a function of UT on January 12 , 1988. Peaks of westward flow, $V_{W}=V_{W P}$ (at an invariant latitude $\Lambda=\Lambda_{p}$ ) are joined by dashed lines. The tick marks give the $\mathrm{V}_{W}=0$ origin for the profile at the UT given by the horizontal axes. Note that the three panels overlap by $5 \mathrm{~min}$. 
(1010-1020 UT) that the eastward flow indicated by the $\mathrm{Ny}$ Alesund magnetometer can be observed in the northerly EISCAT range gates. Fastward flow also appears briefly (at about 0910 UT) between the first and second channels.

Figures $2 c-2 f$ give information on these flow channels. In Figure $2 c$, the gate number (and invariant latitude, $\Lambda_{p}$ ) of the peaks of the flow channels are shown as $\mathrm{P}_{\mathrm{a}}$ function of time. This latitude $\Lambda_{\text {pas }}$ been mapped onto the zenith angle plot in $p_{\text {Figure }} 2 b$, for an assumed emission altitude of $250 \mathrm{~km}$, as shown by the dashed lines. The flow channel and its corresponding 630-nm transient aurora appear to be colocated, at least to within the uncertainty introduced by the assumed emission altitude of $250 \mathrm{~km}$. Note that this is a slightly different location to the 557.7-nm aurora which appeared at the poleward edge of the radar field of view [Iockwood et al., 1989b, Figure 2e]. Lockwood et al. [1989a] and Sandholt et al. [1990] have interpreted this as the 630-nm aurora (wi thout 557.7-nm emissions) arising from the precipitation of sheath like particles down a newly opened flux tube: the 557.7-nm aurora would then be on the poleward edge of this tube, colocated with the upward of the oppositely directed pair of field-aligned currents on the flanks of the newly opened tube which transfer momentum from the magnetopause to the ionosphere in the Southwood model. It can be seen that the flow channels $\left(V_{w}>1 \mathrm{~km} \mathrm{~s}^{-1}\right)$ generally

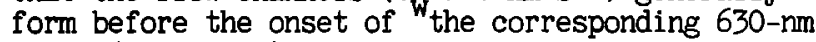
event (Figure $2 \mathrm{~b}$ ), and the two stay at constant latitude (zenith angle) before moving poleward. We cannot tell if the flows persist after the $630-\mathrm{nm}$ event as the corresponding flow channel is by then poleward of the radar field of view. Figure $2 \mathrm{~d}$ shows the value of the peak westward speed, $V_{\text {wp }}$ at the center of the flow channel. It can be wp' seen that this was always largest $\left(2-3 \mathrm{~km} \mathrm{~s}^{-1}\right)$ during the 630-nm event: $V_{\text {wp }}$ was weaker ( 1 - 1.5 $\mathrm{km} \mathrm{s}$ ) before each 630-nm WP event and for events 5 and 8 decreased before the flow channel moved poleward of the field of view toward the end of each transient optical event.

In Figures 2c-2f, the open circles (and thin dashed lines) indicate that the largest $V_{\text {wp }}$ is for the furthest range gate observed (dotted ${ }^{W P}$ line in Figure 3). For these cases, both $V_{\text {wp }}$ and $\Lambda_{p}$ may be underestimates (as $V_{w}$ could peak ${ }^{w p}$ north $p_{\text {of }}$ the radar field of view).

Figures $2 \mathrm{e}$ and $2 \mathrm{f}$ contrast the speed $V_{p}$ of the northward motion of the central peak of $p_{\text {the }}$ westward flow channel (deduced from Figure 2c) and the nor thward component of the derived plasma flow at that peak, $V_{n p}$. Note that, as discussed above, $\mathrm{V}_{\mathrm{np}}$ and $\mathrm{V}_{\mathrm{p}}$ may not be properly determined for

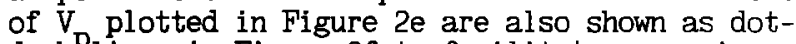

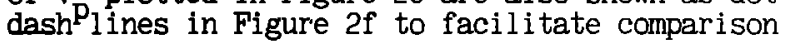
with $\mathrm{V}_{\mathrm{np}}$. In general, the two vary in a similar manner ${ }^{n}$ and have similar magnitudes. For the flow channels to be interpreted as being inside the region of newly opened flux of an FTE, it is necessary that $\mathrm{V}_{\mathrm{np}}$ and $\mathrm{V}_{\mathrm{p}}$ be approximately the same. An obvious ${ }^{n p}$ problem ${ }^{p_{i}}$ the variations in $V_{p}$ is that they can only reflect discrete jumps as $p$ the flow channel usually moves by a whole number of range gates in each 150-s period. To reduce this problem, Figure 4 contrasts the distance $\Delta_{p}$
moved poleward by the flow channel with the

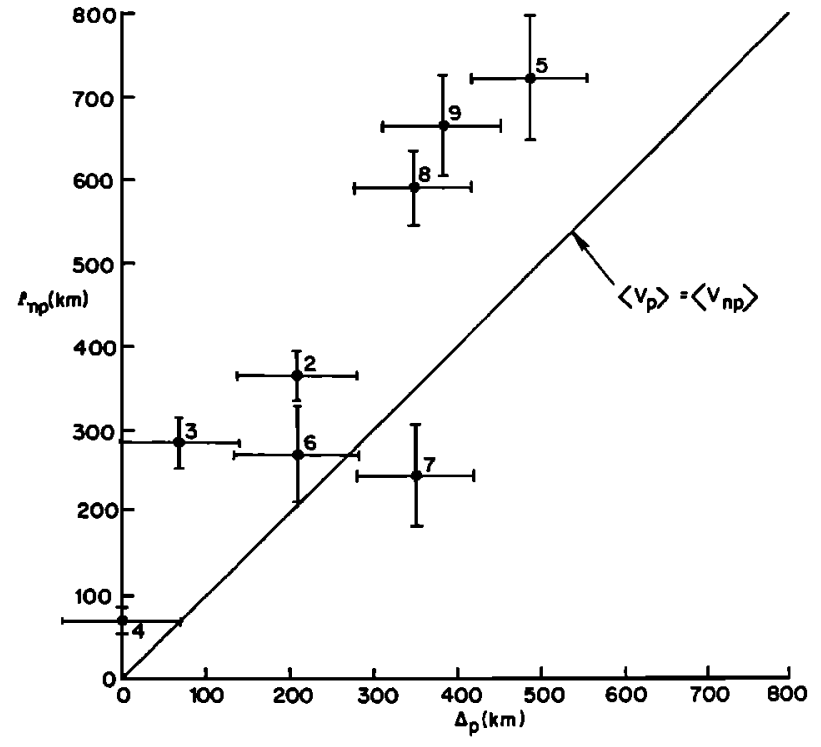

Fig. 4. Distance moved poleward by the flow channels, $\Delta_{\mathrm{p}}$, in their lifetime, T, compared with the value predicted from the observed northward plasma flow within the channel ( $\Delta_{\mathrm{np}}$, as defined by equation (1) of text). Data points are numbered as for the events shown in Figure 2. Note that estimated errors in $\Delta_{n p}$ do not include the unknown errors due to assumptions inherent in the radar beam-swinging technique breaking down.

value deduced from the observed northward plasma speeds, given by:

$$
\Delta_{\mathrm{np}}=\int_{\mathrm{T}} \mathrm{V}_{\mathrm{np}} \mathrm{dt}
$$

during the lifetime, T, of the flow channel. The numbers beside each point refer to the event numbers given in Figure 2a. Errors in $\Delta_{p}$ are calculated assuming that the channel position is known to within half a range gate length at the start and end of its lifetime, and those in $\Delta_{n p}$
reflect the errors in $V_{n p}$ caused by the measurement uncertainty ${ }^{\mathrm{np}_{\text {in }}}$ the line-of-sight velocities (see appendix to Will is et al. [1986]). Hence these errors do not include the effects of the beam-swinging assumptions becoming invalid. It should be noted that the first two data points in event 8 have been excluded. These two points give rise to an equatorward motion of the channel, but only just qualified as a channel on two counts: firstly, $\mathrm{V}$ only just exceeded $1 \mathrm{~km} \mathrm{~s}^{-1}$ (Figure 2d); 'Wp secondly, the peak for the second of the two is very poorly defined (see Figure 3), $V_{w}$ being the same to within $50 \mathrm{~m} \mathrm{~s}^{-1}$ for the first four range gates. However, strictly the periods used in compiling Figure 4 are the poleward moving phases of each event: this only differs from the total channel lifetime, T, for event 8.

The straight line in Figure 4 corresponds to $\left\langle\mathrm{V}_{\mathrm{np}}\right\rangle=\left\langle\mathrm{V}_{\mathrm{p}}\right\rangle$ for the event lifetime. Only for the ${ }^{\text {np events } 4,}, 6$ and 7 are the data consistent with this condition to within the known errors. In general, there is a tendency for $\left\langle\mathrm{V}_{\mathrm{np}_{7}}\right\rangle$ to exceed the value of $\left\langle\mathrm{V}_{\mathrm{p}}\right\rangle:$ all events except ${ }^{n p_{7}}$ and 3 are

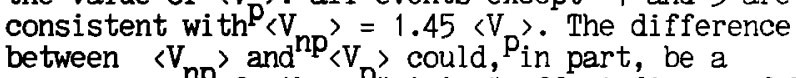
consequence of $^{2}$ the "mixing" effect discussed by Etemadi et al. [1989], whereby spurious northward 
a

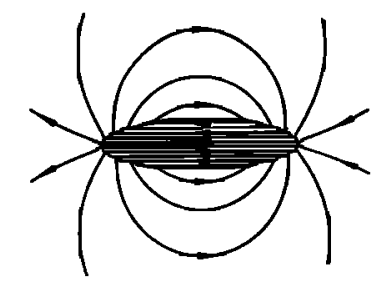

b

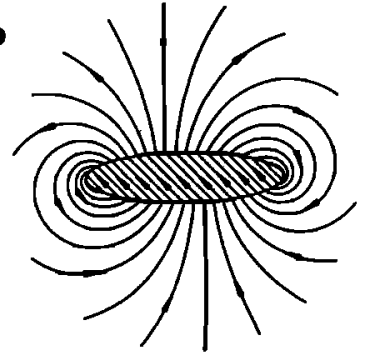

c

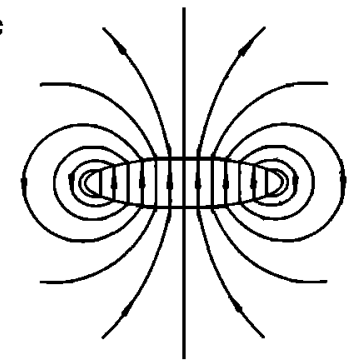

Fig. 5. Flow equipotentials for three phases of motion of an elliptical newly opened flux tube, moving (a) westward at $3 \mathrm{~km} \mathrm{~s}^{-1}$, (b) northwestward at $2 \mathrm{~km} \mathrm{~s} \mathrm{~s}^{-1}$ and (c) northward at $1 \mathrm{~km} \mathrm{~s} \mathrm{~s}^{-1}$. The flux tube major axis is always aligned along the $I$ shell and north is to the top of the Figure. The ratio of the ellipse axes is $b / a=4$.

flows in the radar data are produced by changes in the westward flow. Indeed, the agreement between the speeds $V_{p}$ and $V_{n p}$ (Figures $2 e$ and $2 f$ ) was worst when $v^{p}$ was ${ }^{n p}$ changing rapidly (Figure $2 d$ ). In addition, the flow channel, as given by the peak westward flow, will only be inside the FTE open flux tube when it is moving westward. When it is moving north, the peak westward flows will be northwest and southeast of the open tube (see discussion of Figure 5). The radar will only be able to observe the latter of these due to its limited latitudinal coverage. Hence the observed swing to northward motion of the event will tend to cause the northward motion of the westward flow channel to be smaller than the nor thward FTE motion. With these factors in mind, we consider the agreement of northward event motion and northward plasma flow to be good.

Iockwood et al. [1989a] derived estimates of the event size with the assumption that an open flux tube region was dragged over the radar field of view. In view of the above, more detailed considerations, we here wish to revise these estimates, using the same assumption. Table 1 lists the events seen in full during the period and gives the lifetimes of each flow channel ( $V$ wp $\left.>1 \mathrm{~km} \mathrm{~s}^{-1}\right), \mathrm{T}$, and of the corresponding 630-nm wp transient aurora, $\tau$. The total length of the flow channel, $B_{\text {ing }}$ has been optained by integrating $V_{w p}$ with respect to time during the lifetime, $T$. However, the variations of $V_{\text {p }}$, al lowing for the known smoothing effect of the beamswinging, suggest that the radar is only within the open flux tube, roughly while the photometer observes the enhanced (intensity > $3 \mathrm{kR}$ ) 630-nm emission. This being the case, the enhanced $630-\mathrm{nm}$ emission would arise from the precipitation of magnetosheath plasma down the newly opened flux tube. Integrating $V_{\text {with }}$ wime during these periods (of length $\tau$ ) ${ }^{\mathrm{WP}}$ then yields the best estimates of the east-west dimension of the required open flux tubes, $b$, which are also given in Table 1. In addition, Figure 3 has been used to estimate the peak latitudinal extent of the flow channels, a. The values for a given in Table 1 are the estimated width of the region where $V_{w}$ exceeds $1 \mathrm{~km} \mathrm{~s}^{-1}$. In cases where only the equatorward portion of the flow channel was observed, the peak flow is taken to be at the center of the flow channel, so that the width of the equatorward portion was measured and taken to be $a / 2$. Note that for event $8, a$ is given as $>400$ $\mathrm{km}$, as all range gates give $\mathrm{V}_{\mathrm{w}}>1 \mathrm{~km} \mathrm{~s}^{-1}$.

Table 1 shows that in only one case (event 4) does the event have similar dimensions in the north-south and east-west directions. In general the events are considerably extended in the eastwest dimension (such that $b / a$ is typically 4 ) and the values of $b$ are roughly equivalent to $4-5$

TABLE 1. Dimensions of Transient Flow Burst Events.

\begin{tabular}{|c|c|c|c|c|c|}
\hline $\begin{array}{l}\text { Event } \\
\text { Number } \\
\text { (See } \\
\text { Figure 2) }\end{array}$ & $\begin{array}{l}\text { Lifetime } \\
\text { of Flow } \\
\text { channel, } \\
T, S\end{array}$ & $\begin{array}{l}\text { Lifetime } \\
\text { of } 630-\mathrm{nm} \\
\text { Arc, } \\
\tau, \mathrm{s}\end{array}$ & $\begin{array}{l}a, \\
\mathrm{~km}\end{array}$ & 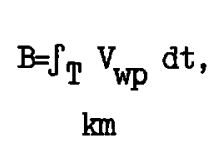 & $b=\int_{\tau} V_{w p} d t$, \\
\hline $\begin{array}{l}2 \\
3 \\
4 \\
5 \\
6 \\
7 \\
8 \\
9\end{array}$ & $\begin{array}{r}750 \\
900 \\
450 \\
1650 \\
1350 \\
1350 \\
1350 \\
1500\end{array}$ & $\begin{array}{l}560 \\
580 \\
525 \\
260 \\
340 \\
695 \\
710 \\
525\end{array}$ & $\begin{array}{r}300 \\
200 \\
350 \\
250 \\
300 \\
350 \\
>400 \\
300\end{array}$ & $\begin{array}{r}1830 \\
2070 \\
384 \\
2347 \\
1810 \\
1986 \\
2230 \\
2310\end{array}$ & $\begin{array}{r}1600 \\
1880 \\
480 \\
1800 \\
1600 \\
1500 \\
1700 \\
1900\end{array}$ \\
\hline
\end{tabular}

Here $a$ is the peak north-south width of flow channels, while $b$ is the best estimate east-west dimension for a convecting flux tube model. 
hours of magnetic local time. The values of a are near $300 \mathrm{~km}$, which roughly maps to a spatial extent of order $2 \mathrm{R}_{\mathrm{F}}$ at the magnetopause, using a typical ratio of the magnetic fields at the dayside magnetopause and in the ionosphere. Hence to within the uncertainties in the mapping factor and in the determination of a $( \pm 50 \mathrm{~km})$ we can say that the latitudinal width of the events is consistent with the typical scale size of an FIE on the magnetopause [Saunders et al., 1984]. The mapping of the east-west dimension of the ionospheric events is even more uncertain. Crooker and Siscoe [1990] have shown that a circular [Russell and Elphic, 1978; 1979] FTE flux tube can map down to an ionospheric region which has large longitudinal extent if the magnetosphere is closed, other than for that tube. If, however, as seems more likely, the FIE is appended to an open polar cap, the circular FTE at the magnetopause maps to a more nearly circular region in the ionosphere [Crooker, 1990]. In this latter case, the values of $b$ inferred here support the Southwood et al. [1988] and Scholer [1988] models of magnetopause FIEs, where the ionospheric projection of the reconnection neutral line covers about 4-5 hours of MLT (and the neutral line may be of order 10-15 $R_{F}$ in length). However, these estimates are highly approximate as the field line mapping from the ionosphere to the magnetosphere is not known in any detail, as discussed above.

\section{Generalized Model Predictions of FTE Signatures}

The combined radar, optical and magnetometer observations reported here and by Lockwood et al. [1989a, b] and Sandholt et al. [1990] give an indication as to why FTE effects have been so elusive in magnetometer data and may explain why reported signatures [Ianzerotti et al., 1987; Bering et al., 1988] are not in complete agreement with the models. The modeling of the magnetic perturbations seen by a single station was carried out by McHenry and Clauer [1987] and is based on the two models shown in Figures $1 \mathrm{a}$ and $1 \mathrm{~b}$ but, as discussed earlier, there are a number of important assumptions: that the ionospheric conductivities are uni form and constant; that the events have a constant velocity, $\mathrm{v}$, and are circular in cross sention; that an event occurs in isolation; and that during the ionospheric signature's if etime it will pass completely through the region where the magnetometer is sensitive to the ionospheric currents. All of these assumptions must be questioned in the light of the observations discussed in this paper. The last of them has implications for the detection rate of FIEs in the ionosphere, as well as for the nature of the FTE signature. The lifetime of transient dayside aurorae is found to be typically 2-15 min. This is consistent with the predictions for the flow and current signatures of FTEs by Lockwood et al. [1990], based on the delays in the responses of ionospheric flows to changes in the IMF. As pointed out by Iockwood and Cowley [1988], this means that the probability of observing an event at any one ground observatory is very much smaller than that for a satellite at the magnetopause. Sandholt et al. [1990] have shown that westward moving events have highly enhanced ionospheric conductivities close to their poleward boundary, where a strong 557.7-nm arc is observed. This arc has been associated with the upward field-aligned current of the oppositely directed pair required to transfer the momentum from the magnetopause to the ionosphere in the Southwood FWE model

[Lockwood et al., 1989a; Sandholt et al., 1990], and the current associated with it dominates the observed impulsive magnetometer signatures.

In addition to the above considerations, Table 1 indicates that these events are far from being circular. In this section we generalize the model predictions for a Southwood FIE model from the circular flux tube shown in Figure la to one of elliptical cross section. We only predict the F region flows which would be observed by a radar, thus avoiding some of the conductivity complications discussed above which must surely significantly alter the signatures in data from ground-based magnetometers. However, we note that the observed pattern of motion which we input into the model may well be influenced by the patch of enhanced conductivity which will move with that part of the event. A fully self-consistent treatment of the signature would not be able to neglect this effect. Note that a long and thin patch of enhanced conductivity plasma, with enhanced flow along it, is an electrojet and is analogous to those observed on the nightside during substorms.

In Figure 5, we consider the flow in and around a moving elliptical newly opened flux tube in the northern hemisphere. From Table 1, we take the ratio of the lengths of the major and minor axes to be 4 , with the major axis always aligned along an $\mathrm{L}$ shell, as inferred from the all-sky TV camera images of these events [Sandholt et al., 1990]. For these illustrative patterns the shape, area and axis ratio of the flux tube are all taken here to remain constant. In general, the area of such a tube will grow while reconnection proceeds (according to Faraday's law): the shape may well also evolve with time. In addition, we here do not consider any untwisting of the flux tube. The patterns are derived using the standard hydrodynamic equations of incompressible flow around an elliptical obstacle [e.g. MilneThompson, 1955]. The flow speeds and directions used are taken from the pattern of event motions described in the previous section.

Subsequent to submitting this paper we have become aware of a paper by Wei and Lee [1990] who have derived the flow and field-aligned current patterns for moving elongated plasma clouds. Their results agree with those presented in Figure 5. In fact, they present flow patterns for the same angles between the direction of motion and the major axis of the ellipse as in Figure 5. Our results are different only in that we have decreased the speed of motion as this angle increases, consistent with the observed pattern of motion of the dayside breakup events. We also point out from these observations and the predicted behavior of newly opened flux tubes that the flow patterns in the ionosphere will evolve between the forms given by Wei and Lee and in Figure 5.

Figure $5 \mathrm{a}$ shows the flow equipotentials (streamlines) for such a flux tube moving westward at $3 \mathrm{~km} \mathrm{~s} \mathrm{~s}^{-1}$, typical of the initial phases of the events discussed in the previous section. In this phase, the flux tube is moving under the dominant 
influence of magnetic tension; the IMF $B_{\text {}}$ component would therefore be positive forl this case. It can be seen that the eastward flow outside the westward moving tube is very weak (large separation of flow equipotentials). This is a feature of many reported FTE signatures. For example, Lockwood and Smith [1989] were able to qualitatively model the flows around a putative FTE observed by the $\mathrm{DE} 2$ satellite, using a circular flux tube model. However, quantitatively, the eastward flows outside the westward moving event were too small and the authors listed several possible reasons for this, including the assumed shape of the flux tube. In general, the ratio of the flow speed inside the event to the peak value outside the event flank is equal to the ratio $b / a$ for an elliptical flux tube. Figure $5 a$ also shows that a station which lies close to the path of the event center will observe westward flow outside the flux tube for a much shorter period, relative to the overall event duration, than for a circular flux tube (compare with Figure 1a). Consequently, as invoked previously, a larger part of the observed westward flow is within the flux tube if it is elongated in the direction of motion than is the case if it is circular.

In part Figure 5b, the event is considered to have slowed to $2 \mathrm{~km} \mathrm{~s}^{-1}$ and is moving

nor thwestward. The change in the direction of motion, relative to the major axis of the ellipse, causes dramatic changes in the twin vortical flow pattern. The strongest flows are now outside the flux tube and in a southeasterly direction.

Finally, in Figure $5 \mathrm{c}$, the event is moving due northward and has further slowed to $1 \mathrm{~km} \mathrm{~s}^{-1}$. In this phase, the dominant influence on the motion is the antisolar flow of that part of the flux tube within the magnetosheath. The peak flows are again outside the open tube (and are now directed southward). As mentioned earlier, westward flow channels are now northwest and southeast of the FTE flux tube.

These calculations show that, even without changing the shape of or twisting the flux tube, the general zonal-then-poleward motion of elliptical newly opened flux tubes described by Lockwood et al. [1989a] and Sandholt et al. [1990] will result in a rapidly evolving large-scale twin vortex pattern.

\section{EISCAT Observations of an Event on January 15, 1988}

From the preceding sections, we infer that the observations on 12 January, 1988 are consistent with the convecting FTE model, if elongated newly opened flux tubes moved westward (for $\mathrm{IMF} \mathrm{B}_{\mathrm{y}}>0$ ) through the radar field of view. In this section we present data from 3 days later, consistent with an eastward moving, near-circular

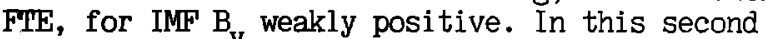
case the radar detects not only a main flow burst, but also a "return" flow vortex, thought to be outside the newly opened flux tube.

On January 15, 1988, EISCAT was also making observations in its Common Programme CP-4 mode. Cloudy skies unfortunately prevented simultaneous optical observations. Figure 6 a gives the flow vectors derived on this day between 09 and 13 UT ( $\approx 12-16 \mathrm{MHT})$, using the beam-swinging technique.

$$
\begin{aligned}
& \text { EISCAT CP-4-A } \\
& \text { January } 15,1988
\end{aligned}
$$

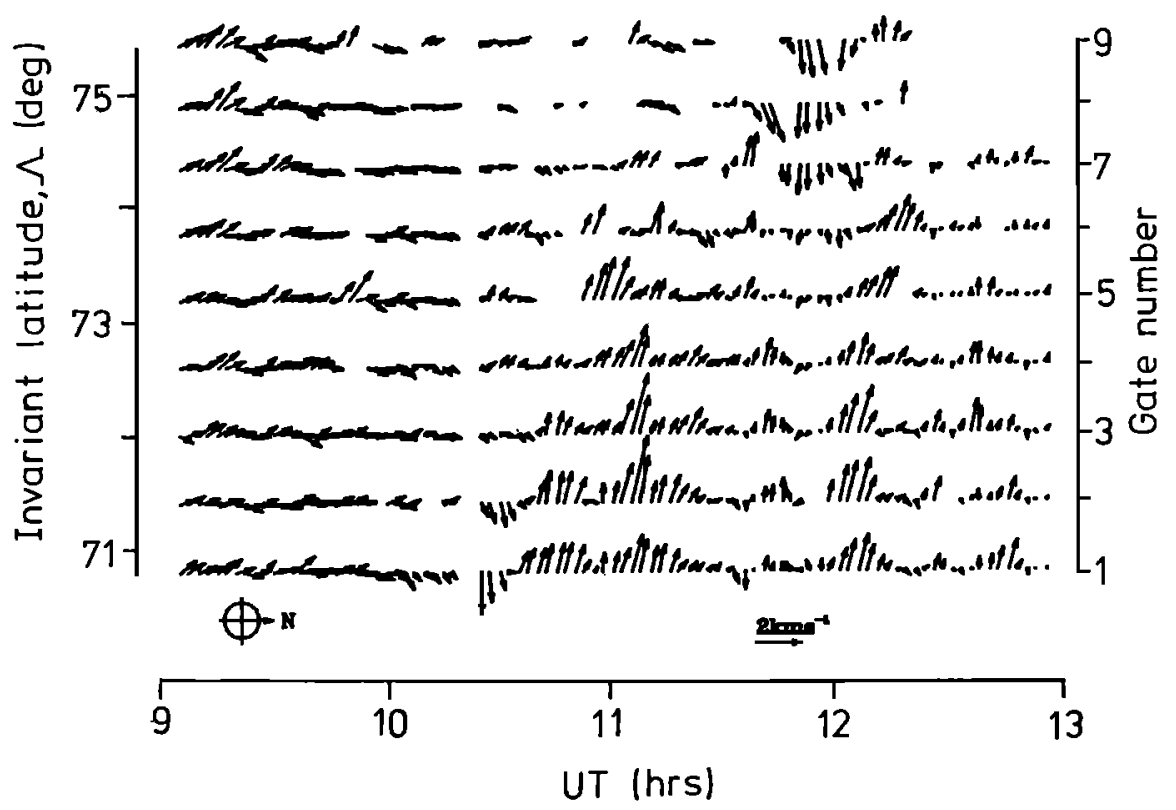

Fig. 6a. Flow vectors observed by EISCAT on January 15, 1988. Flows are shown as a function of invariant latitude and UT, and vectors have been rotated through $90^{\circ}$ to avoid congestion of the plot, i.e., northward flows are depicted by vectors pointing to the right and westward flows by those pointing to the top of the Figure. 


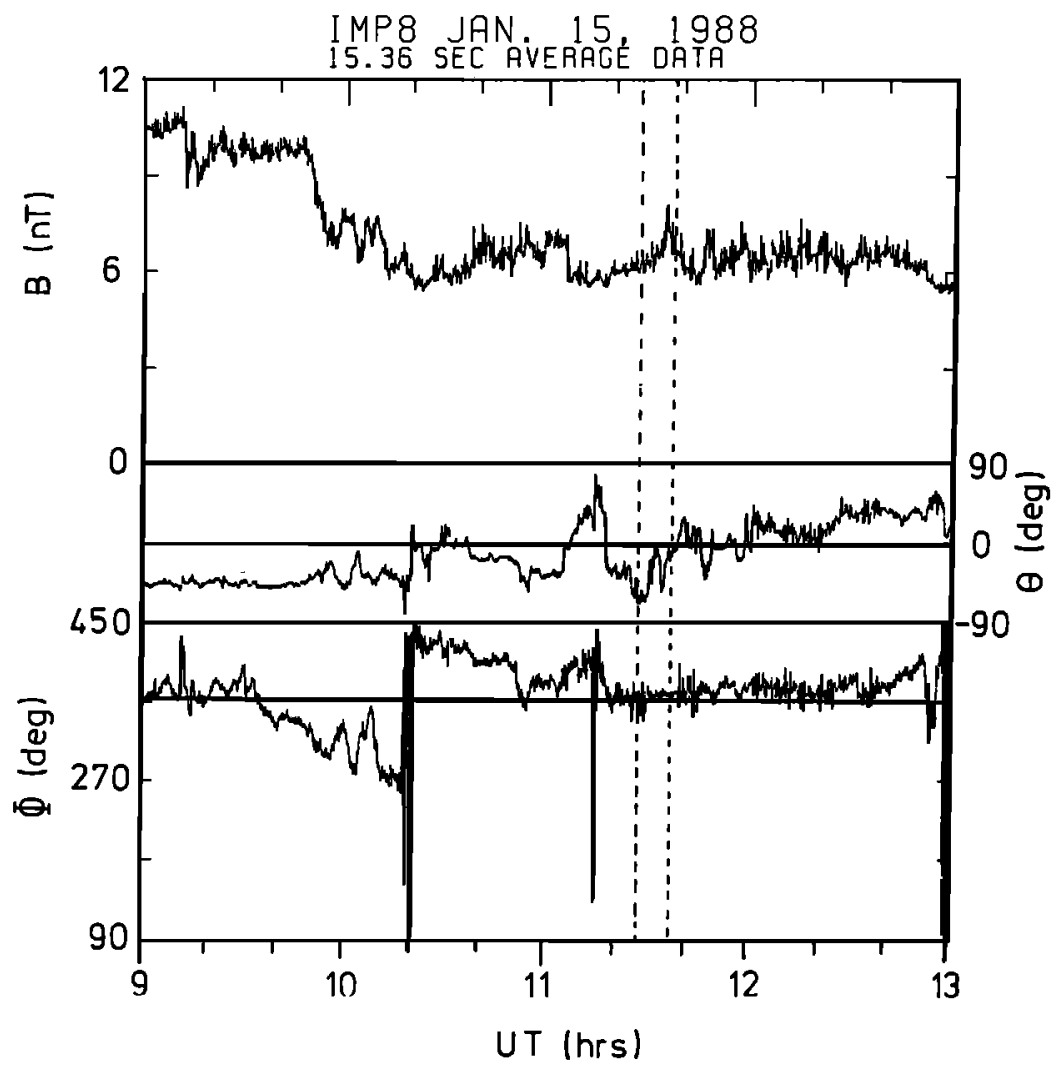

Fig. 6b. The strength and orientation of the IMF (GSM ccordinates) observed by IMP 8.

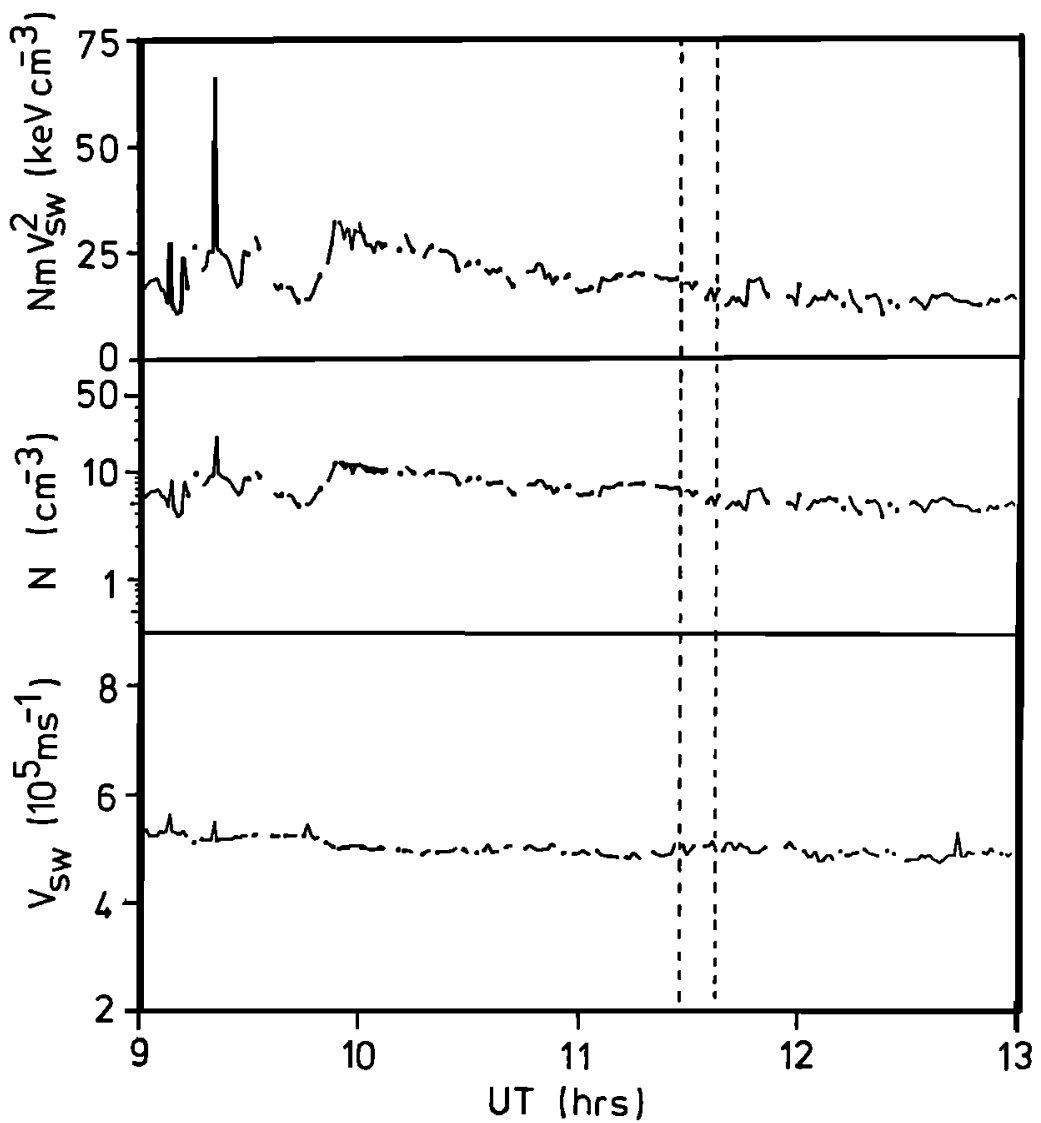

Fig. 6c. The solar wind speed, density and dynamic pressure observed by IMP 8 . 
To avoid congestion of the plot the flow vectors have been rotated clockwise by $90^{\circ}$, so northward flows are shown as pointing to the right of the figure and westward flows to the top of the figure. Figure $6 \mathrm{~b}$ and $6 \mathrm{c}$, show the IMF and solar wind flow observed by the IMP 8 spacecraft at GSE coordinates $\mathrm{X}=29 \mathrm{R}_{\mathrm{F}}, \mathrm{Y}=5 \mathrm{R}_{\mathrm{F}}$ and $\mathrm{Z}=10 \mathrm{R}_{\mathrm{F}}$. The IMF data are given $\mathrm{E}_{\text {in }} \mathrm{GSM}^{\mathrm{E}}$ coordinates $\mathrm{E}^{\text {. Ts the }}$ magnitude, $B$, elevational angle, $\theta$, and azimuthal angle, $\varphi$, of the IMF vector in CSM coordinates. Because $\varphi$ is close to $360^{\circ}$ for the period of interest, it has been plotted on a scale between $90^{\circ}$ and $450^{\circ}$.

Initially (09-1030 UT), EISCAT observes relatively uniform northward flow at speeds of about $1 \mathrm{~km} \mathrm{~s} \mathrm{~s}^{-1}$. At these times, the "Polar" field of view is at an MLT of about 1130-1300 and usually contains little flow (i.e., the field of view is between the flow cells at subauroral latitudes) or westward flow (in the afternoon sector auroral oval) [Willis et al., 1986]. Westward flows did appear in range gate 1 at 1030 UT. This change then propagated poleward, reaching range gate 6 at 1100 UT, and may be associated with the gradual decline in solar wind dynamic pressure seen shortiy before by IMP 8 , but is more likely the result of the sudden change in the IMF from strongly southward $(\theta<0)$ to near zero $B_{z}$ $(\theta \approx 0)$ which occurred at 1018 UT (i.e., the flows within the radar field of view respond 12 min after the changes seen by IMP 8). The IMF change is seen as an increase in $\theta$ to near $0^{\circ}$ which is accompanied by a change in $\varphi$ from $270^{\circ}$ to $450^{\circ}$ (i.e., IMF $B_{\text {swings from positive to }}$ negative). If we $\mathrm{y}^{\mathrm{interpret}}$ this change in ionospheric flows as the convection reversal boundary in the "throat region" (i.e., the poleward flows are in the convection polar cap, whereas the westward flows are in the auroral oval), we find that such a boundary lies between gates 7 and 8 after 1100 UT. Following this, a burst of eastward flow is observed in gates 5-9, between about 1140 and 1210 UT. This event straddles the old convection boundary and is the subject of this section.

This observed propagation delay from the satellite to the radar of $12 \mathrm{~min}$ is very close to the $11.5 \mathrm{~min}$ predicted for this time using the equations given by Farrugia et al. [1989] (also given by Lockwood et al. [1989b]) from the observed solar wind speed, direction and density. At this time the IMF was close to being transverse to the $X$ axis and the solar wind flow; however, shortly before the ionospheric flow event, the IMF adopted a near-radial orientation. If any change in the solar wind dynamic pressure were the cause of this event, the lag could be the $12 \mathrm{~min}$ discussed above if the structure were aligned perpendicular to the solar wind flow. However, the structure may be aligned with the IMF, which has a near-radial orientation. For this second case the propagation delay is very difficult to compute, as the structure will not impinge upon the subsolar magnetopause, as assumed in the Farrugia et al. equations. The propagation delay between the IMP 8 observation and the structure impinging upon the magnetopause will be lower: in fact, we estimate that the structure may have impinged upon the magnetopause up to $5 \mathrm{~min}$ before it would be seen at IMP 8, which means an ionospheric signature could have been present 3 minutes before its trigger was seen at IMP 8. However, the point of impact, and hence the initial ionospheric signature, would be in the dawn sector (from the above calculation at about $8 \mathrm{MLT}$ ), and thus there will be a delay for the perturbation to propagate eastward to the radar which was at 1430 MLT at the start of the event. An ionospheric dynamic pressure signature is predicted to propagate at about $6 \mathrm{~km} \mathrm{~s}^{-1}$ (see introduction), which gives an additional delay of $5.5 \mathrm{~min}$. (In fact we will later show that the ionospheric event moves eastward over the radar at the much lower speed of $1.1 \mathrm{~km} \mathrm{~s}^{-1}$ ). Hence we would estimate the delay to be $2.5 \mathrm{~min}$, if the solar wind structure is aligned with the IMF. Hence we would expect any event trigger to have been observed by IMP 8 between 1128 and 1137:30 UT?. (the dashed lines in Figures $6 \mathrm{~b}$ and $6 \mathrm{c}$ ).

Within this interval there are two pulses of strongly southward IMF (decrease in $\theta$ ), with a $B_{4}$ weakly negative, but close to zero. However, the solar wind dynamic pressure had been constant prior to this interval and showed a gradual decline (by $35 \%$ ) over the subsequent $20 \mathrm{~min}$. Also within this interval we note a spike in IMF magnitude, $B$, at 1135 UT. We will later discuss how an increase in $B$ could imply a drop in solar wind dynamic pressure. In fact, assuming pressure equilibrium in an isothermal solar wind and using electron and ion temperature of $10^{5} \mathrm{~K}$ and $2.4 \mathrm{x}$ $10^{4} \mathrm{~K}$, respectively (as derived by Burlaga [1968] for such pressure equilibrium regions), one would predict a decrease in dynamic pressure of $2.7 \mathrm{nPa}$. The solar wind data sequence is interupted by two short ( $2 \mathrm{~min}$ ) data gaps near the peak in $B$; nevertheless, at the time of the maximum $B$ there were observations, and the dynamic pressure is only $0.4 \mathrm{nPa}$ lower than at the onset of the rise in $\mathrm{B}$ and is actually $0.2 \mathrm{nPa}$ higher than at the end of the fall in B. We conclude that the use of IMF $B$ to quantify dynamic pressure changes must be treated with great caution, either because of the assumptions about pressure equilibrium and/or constant temperatures, or because of the need to assume ion and electron temperature values.

Hence within the predicted period for a trigger there are two swings to strongly southward IMF, but no dynamic pressure changes which are any larger than the fluctuation level observed throughout the period shown in Figure 6 . We do note, however, that at 1147 UT there is a dynamic pressure pulse which, although small in absolute terms $(0.8 \mathrm{nPa})$, does represent a $50 \%$ increase. However, this would appear to be too late to generate the ionospheric event (eastward flows commenced in gate 8 at 1140 UT). The first effects of this pulse would be expected in the ionosphere at about 1144 UT in the dawn sector, and effects at the MUT of EISCAT would not be expected until after about $1150 \mathrm{UT}$; whereas the observed event comenced at 1140 UT. Hence the solar wind dynamic pressure does not show a suitable trigger for the eastward flow event seen by EISCAT, but there is a pulse which could have influenced the second half of the event (after 1150 UT). It should be noted that the IMF is near radial, and it has been suggested that pressure pulses in the magnetosheath may form under such conditions, even for uniform solar wind flow [Fairfield et al., 1990]. The IMF does show two strong swings to southward, which is the favored orientation for 
FTE occurrence, in the interval when an event trigger is experted.

Interpretation of these flow vectors in terms of the vortical flow patterns shown in Figure 1 is difficult, because the usual care must be taken to eliminate spurious vectors due to the use of the beam-swinging technique in the presence of the predicted spatial gradients and temporal changes of the flow. However, in the center of the observed eastward flows we find little variation from gate to gate and from data point to data point: hence we can have some confidence in these vectors. However, nearer the edges of this region large shears/changes in the flow are observed, and the vectors will undoubtedly be in error to some extent. Bearing this in mind, we here place particular emphasis on the ion temperature observed at the two azimuths. When drifts are large, ion-neutral frictional heating dominates the energy balence equation of the ion gas, which then becomes [St.-Maurice and Hanson, 1982]

$$
\mathrm{T}_{\mathrm{i}} \approx \mathrm{T}_{\mathrm{n}}+\mathrm{m}_{\mathrm{n}}|\underline{\mathrm{V}}-\underline{\mathrm{U}}|^{2} / 3 \mathrm{k}
$$

where $\mathrm{T}_{j}$ is the average, three-dimensional value of ${ }^{1}$ the ion temperature, $T_{n}$ is the neutral temperature, $\mathrm{m}$ is the neutral mass, $\mathrm{V}$ is the bulk velocity of the ion gas, $U$ is the neutral wind velocity and $k$ is Boltzmann's constant. In fact, the temperatures presented here are derived with the assumption that the ion gas has a Maxwellian distribution of velocities, giving values we term here as $\mathrm{T}_{\text {. }}$. When the velocity difference $|\underline{V}-\underline{U}|$ is large, the ion velocity distribution function becomes anisotropic and tends toward a toroiodal form [St.-Maurice and Schunk, 1979; Iockwood and Winser, 1988]. For the large aspect angles with which the plasma is viewed by the $\mathrm{CP}-4$ experiment, this will cause $\mathrm{T}_{\mathbf{i m}}$ to be an overestimate of the real ion temperature $\mathrm{T}_{i}$; however, $\mathrm{T}_{i m}$ will always increase monotonically with the real im $_{i}$ temperature, $\mathrm{T}_{j}$ [Suvanto et al., 1989; Lockwood and Winser, 1988]. Hence $T_{i m}$ can be used to define any spatial and temporal ${ }^{1 m}$ changes in $|\mathrm{V}-\mathrm{U}|$. In addition, if temporal changes in $V$ take place on time scales short compared with the time constant for changes in $U$ (typically 10 min - 1 hour), we can use $T_{i m}$ to Identify changes in $\mathrm{V}$. This technique has previously been employe $\bar{d}$ with Polar data by Lockwood et al. [1986] to identify the eastward propagation of a convection enhancement following a southward turning of the IMF, and by Lockwood et al. [1988] to determine the orientation of a moving shear flow reversal. Because $T_{\text {im }}$ is a scalar quantity, Polar can be viewed as making independent observations at two points on each $I$ shell studied, the separation of these two points increasing from $200 \mathrm{~km}$ for gate $1 \mathrm{up}$ to $400 \mathrm{~km}$ for gate 8 .

In addition, we employ the technique described by Todd et al. [1988] to examine the 10-s line-ofsight velocities observed at each azimuth. This enables evaluation of some assumptions made in the vector derivation. However, 10-second data are only available for the nearer range gates for which signal/noise ratios are largest.

Figure $7 \mathrm{a}$ shows the temperature, $\mathrm{T}_{\mathrm{im}}$, for gate 3 during the period when the eastward ${ }_{\text {flow burst }}$ event is observed in gates 7-9. The solid line and solid circles are for azimuth $i\left(12^{\circ}\right.$ to the west

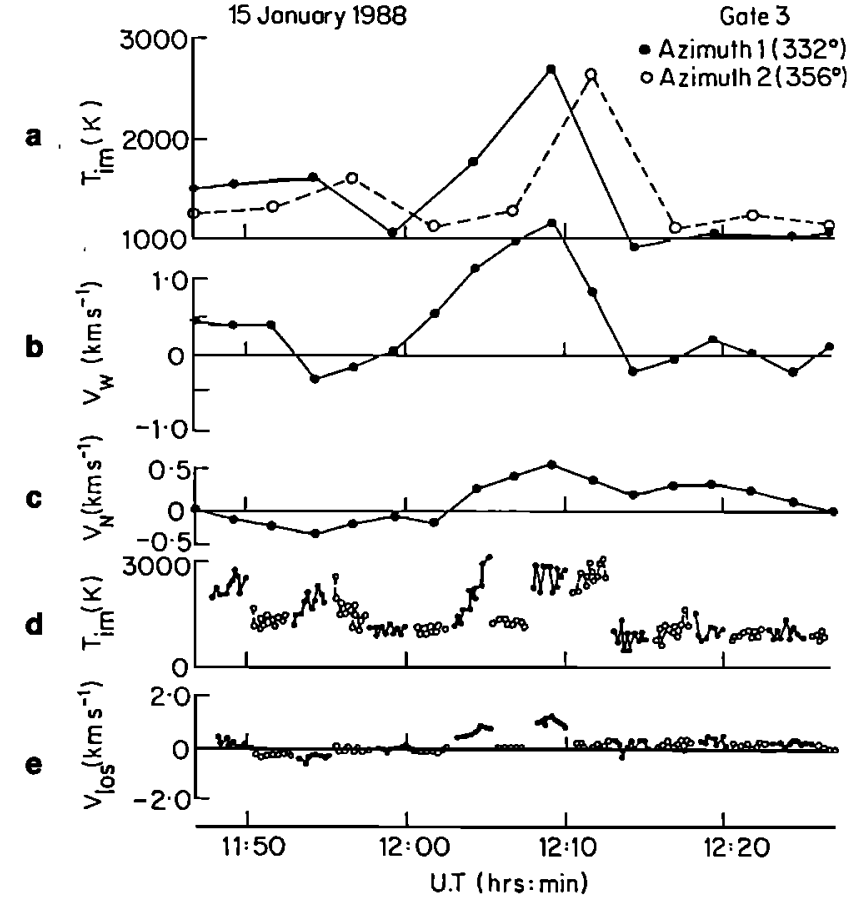

Fig. 7. Flows and ion temperatures for gate 3 observed by EISCAT on January 15, 1988. Solid circles and lines are for azimuth 1; open circles and dashed lines are for azimuth 2. (a) The ion temperatures $\mathrm{T}_{\mathrm{im}}$ derived assuming a Maxwellian ion velocity distribution function from 2-min postintegrations of the data. (b) and (c) The westward and northward flow components derived from the beanswinging technique. (d) The 10-s integrations of $\mathrm{T}_{\mathrm{im}}$. (e) The line-of-sight velocities at $10-\mathrm{s}$ resolution.

of the I shell meridian), the open circles and dashed line are for azimuth $2\left(12^{\circ}\right.$ to the east). These data are postintegrated over the 2-min dwells of the beam at each azimuth. Analysis allowing for the non-Maxwellian nature of the ion gas under strong drift conditions has alsc been carried out, using the analysis procedure described by Suvanto et al. [1989]. The nonMaxwellian nature of the plasma is then quantified by a shape distortion parameter, $D^{*}$. In Figure 7 , we only show the Maxwellian analysis because data for further range gates or lower postintegration times have insufficient signal/noise ratios to allow non-Maxwellian analysis. We find $D^{*}$ cannot be distinguished from a zero (the Maxwellian limit) at all times for gate 3 , except when $\mathrm{T}_{\text {im }}$ peaks. Then $D^{*}$ reaches 0.8 (by way of comparison, $D^{*}=1.2$ is the threshold for a toroidal distribution), and $\mathrm{T}_{\mathrm{i}}$ is found to be $2700 \mathrm{~K}$, slightly lower than ${ }^{T_{1 m}}$ wich is close to $3000 \mathrm{~K}$.

Figure $7 d$ gives the results for $T_{i m}$ from 10-s integrations of the same data, and Figure $7 e$ shows the corresponding line-of-sight velocities. The latter show a relatively smooth variation, with good consistency between adjacent data points. Using the interpretation rules given by Todd et al. [1988], the line-of-sight velocities indicate weak southward flow around 1153 UT and strong northwest flow around the $\mathrm{T}_{i m}$ peaks near 1200 UT. The line-of-sight data 
therefore give us some confidence in the flow components derived from the beam-swinging technique, which are shown in panels Figures $7 \mathrm{~b}$ and $7 \mathrm{c}$.

Figure 7a shows that very similar variations in $\mathrm{T}_{\text {im }}$ are observed in both azimuths, but that for azimuth 2 lags that for azimuth 1 by about $2.5 \mathrm{~min}$. In other words, the ion temperature enhancement (and hence the enhanced westward flows that caused it) is moving eastward across the Polar field of view. From data of the resolution of those in Figure $7 \mathrm{a}$ we can only say that the lag is in the range $0-7.5 \mathrm{~min}$ and we must address the higher resolution data (Figure 7d). A sharp rise in $T_{\text {im }}$ is observed at azimuth 1 between 1203:30 UT and 1204:50 UT. This rise occurred at azimuth 2 at some time between 1207:30 and 12:10:30 UT (while the antenna was at azimuth 1 or in motion), and hence the lag is in the range 220$300 \mathrm{~s}$. The subsequent fall in $\mathrm{T}_{\text {im }}$ occurs in the periods 1210:00 - 1213:00 JT imd 1212:30 1215:30 UT for azimuths 1 and 2 respectively. This limits the lag to the range -30 to $330 \mathrm{~s}$, consistent with that found for the rise in $\mathrm{T}_{\text {im }}$. The best estimate of the lag is therefore $260 \pm 40 \mathrm{~s}$. As the separation of the centers of the two scattering volumes is $280 \mathrm{~km}$ for gate 3 , the above lag quantifies the speed of eastward motion of the region of westward plasma flow as $v_{e}=$ $1.1 \pm 0.2 \mathrm{~km} \mathrm{~s}^{-1}$.

Figure 8 is the same as Figure 7, but for gate 7. Because insufficient signal/noise ratio was obtained for this gate, the data cannot be analyzed at 10-s resolution and hence the panels corresponding to Figures $7 \mathrm{~d}$ and $7 \mathrm{e}$ are absent. Again, a similar variation in $T_{i m}$ is observed at the two azimuths, and values for ${ }^{1 m}$ azimuth 2 consistently lag those for azimuth 1. For these lower resolution data, we can only say the lag is consistent with the eastward speed of $1.1 \mathrm{~km} \mathrm{~s}^{-1}$ derived for the motion of the westward flow region over gate 3 . This speed is also very similar to the derived eastward flow speed within the burst seen in gate 7 (see Figure $8 b$ ). That the variations of $T_{i m}, V_{n}$ and $V_{w}$ seen by
the radar during this event
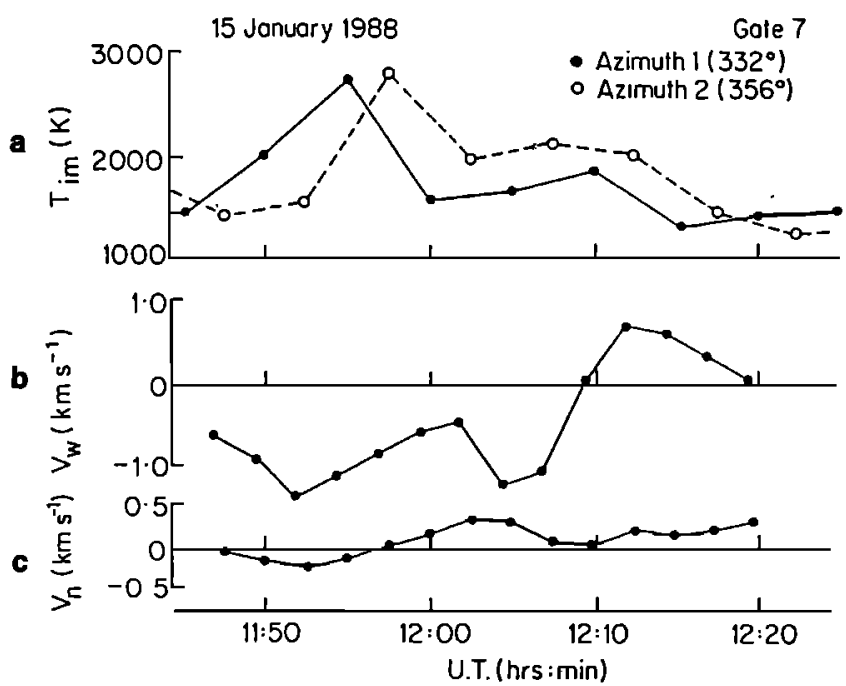

Fig. 8. Same as Figures $7 \mathrm{a}, 7 \mathrm{~b}$ and $7 \mathrm{c}$, for the same interval and gate 7 . consistent with a convecting FIE model is demorstrated by Figure 9. This does not constitute an atterut to fit the data as there are many free variables (axis ratio of ellipse, orientation of ellipse, area of ellipse, distance of closest approach to radar, direction and speed of motion, neutral temperature, neutral wind) and invalid assumptions (mainly that event velocity, $\mathrm{v}_{e}$, remains constant). Nonetheless, comparison of Figures 7, 8 and 9 demonstrates that the temperature and flow data are well explained by an eastward moving convecting FTE signature. The top of the Figure shows the model open flux tube employed, with north to the top of the Figure, in its own rest frame. The elliptical flux tube has major and minor axes lengths of $b=600 \mathrm{~km}$ and $a=$ $400 \mathrm{~km}$, and the major axis is inclined at $25^{\circ}$ to
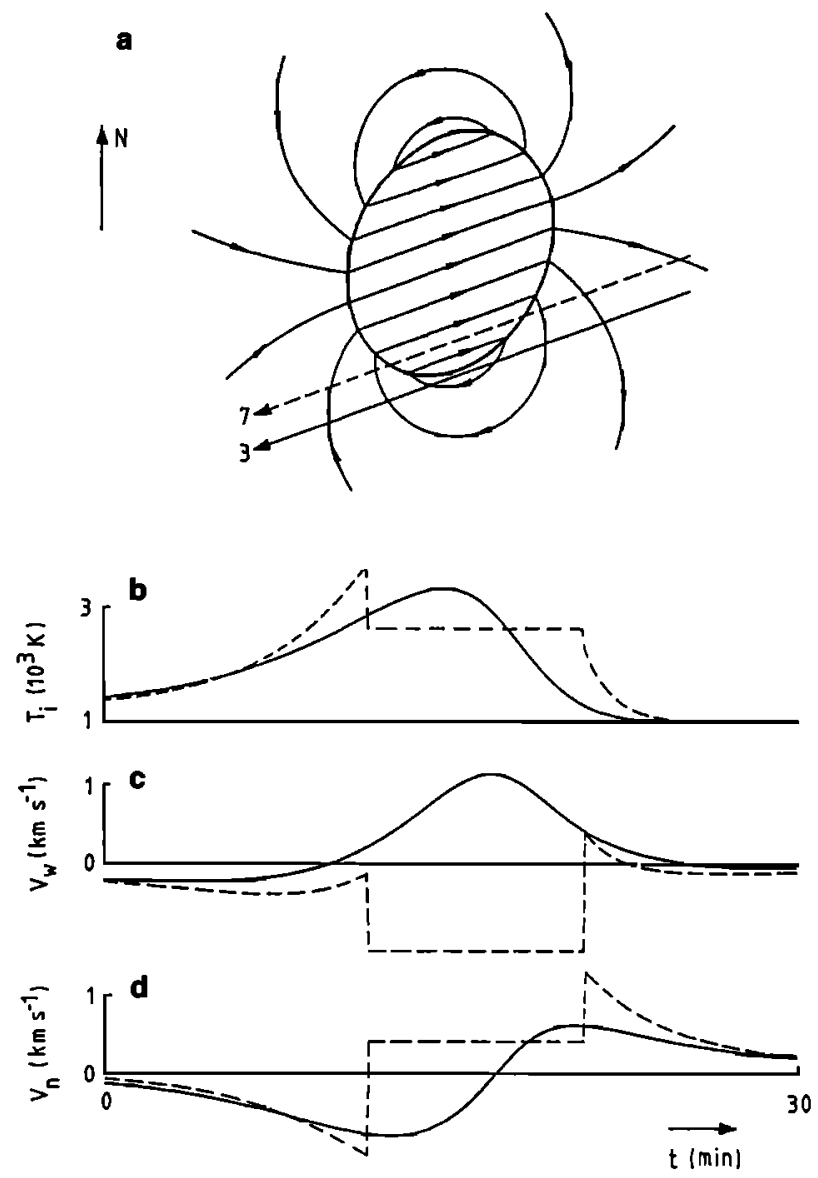

Fig. 9. (a) Model equipotentials around an elliptical Southwood FTE flux tube, shown in the rest frame of the tube. In this frame the loci of gates 3 and 7 are shown as solid and dashed lines for a 30-min intersection period. The event velocity is $\mathrm{v}_{\mathrm{e}}=1.2 \mathrm{~km} \mathrm{~s}$ at all times and directed $20^{\circ}$ north of east. The flux tube axes are $a=400 \mathrm{~km}$ and $b=600 \mathrm{~km}$, and the major axis is at an angle of $25^{\circ}$ east of north. The lower three panels show the variations of parameters observed in the half-hour intersection period by gates 3 and 7 (solid and dashed lines, respectively): (b) the ion temperature (for $m_{n}=16 \mathrm{amu}, U=400 \mathrm{~m}$ $\mathrm{s}^{-1}$ nor thward and $\left.\mathrm{T}_{n}=1000 \mathrm{~K}\right),(\mathrm{c})$ the westward plasma flow, $V_{W}$, and (d) the northward plasma flow, $V_{n}$. 
the northward direction. The event is moving at $1.2 \mathrm{~km} \mathrm{~s}$ in a dijection $20^{\circ}$ north of east: hence the eastward component of motion is $1.1 \mathrm{~km} \mathrm{~s} \mathrm{~s}^{-1}$, as for the observations. The flows in the reference frame of the tube are shown by the equipotentials marked with arrows, and the solid and dashed lines are the loci of radar gates 3 and 7 for a halfhour period. The variations of the ion temperature and flow components at these two range gates (strictly, for the point midway between the two scattering volumes for the two beam directions) during this period are shown in the three lower panels (with solid and dashed lines for gates 3 and 7 , respectively). The ion temperature is computed from equation (2) assuming that $U$ is northward with a speed of $400 \mathrm{~m} \mathrm{~s}^{-\mathrm{P}}, \mathrm{T}_{\mathrm{n}}$ is $1000 \mathrm{~K}$ and $\mathrm{m}_{\mathrm{h}}$ is $16 \mathrm{amu}$ (0 atoms).

Many of the features of the observations are well reproduced in this simulation. For example, the ion temperature peaks first for the further range gate (near $3000 \mathrm{~K}$ ) but then remains near $2000 \mathrm{~K}$ (when within the newly opened tube) until just after the temperature falls in the nearer gate. The derived vectors also show remarkably similar variations to those from the simulations (considering the uncertainties already discussed). Some features cannot be reproduced. For example, the data show very slow flows in gate 3 near 1200 UT, whereas the model predicts that flow at this time should have been southward, a difference which can be explained by the limitations of the beam-swinging technique as the event approaches the radar. Similarly, we would expect the derived vectors to underestimate the northward flow (and exaggerate the westward flow) for gate 7 when it exits the open flux tube.

The limitations of the beam-swinging technique do not allow us to fit the sequence exactly; however, the ion temperatures (which are not subject to this error) are well explained by the model. The ion temperature data do show that the perturbation in all range gates is moving eastward at around $1 \mathrm{~km} \mathrm{~s} \mathbf{s}^{-1}$.

\section{Discussion of Flow Bursts on January 12, 1988}

The data for January 12, 1988, reveal a series of westward flow channels forming immediately equatorward of the cleft/cusp aurora. Fach is associated with a transient auroral event observed in both 630-nm and 557.7-nm emissions which form immediately equatorward of the persistent cleft aurora and fade several degrees poleward of it. Peak westward flows of $3 \mathrm{~km} \mathrm{~s}$ are observed, and the flow channels systematically move poleward. The flow burst/transient aurora events are found to recur every $8 \mathrm{~min}$ if the IMF $B_{3}$ component is continuously southward or following isolated swings to southward IMF [Lockwood et al., 1989b] . The transient aurorae have been shown to move with the ExB/B $B^{2}$ drift of the local plasma, and both initially move westward and slow as the motion continuously evolves to poleward.

Lockwood et al. [1989b], in their Figure 7, sketched the shape, flows and pattern of motion for these events. In view of the dimensions $a$ and $b$ derived in Table 1, this schematic should appear as in Figure 10. Note that the events could now be described as a "peeling off" of closed field lines over a large portion of the afternoon sector

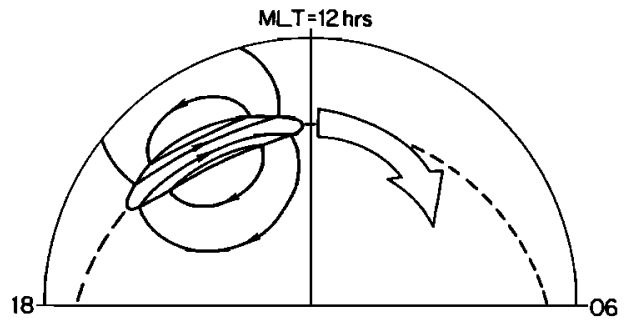

Fig. 10. Schematic of typical event observed on January 12, 1988. The region of newly opened flux and flows are shown early in their lifetime, and the large arrow gives the subsequent motion of the event.

auroral oval (covering typically 4-5 hours of MLT) which subsequently move west around the polar cap boundary (for strongly positive IMF $B$ in the northern hemisphere) and then poleward $\mathrm{y}_{\text {into }}$ the polar cap.

We believe that these transient events reflect transient momentum transfer across the magnetopause. We know of no theory that suggests that such large flows in the cusp/cleft region can be generated internally by the magnetosphereionosphere system. Here we discuss the two proposed mechanisms for such momentum transfer across the magnetopause and into the ionosphere discussed in the introduction: transient reconnection ("flux transfer events") [Southwood 1985, 1987; Cowley, 1986], and dynamic pressure changes [Southwood and Kivelson, 1990; Sibeck et al., 1989a, b, c; Lee, submitted, 1990].

\section{Interpretation in Terms of Dynamic Pressure Effects}

As discussed earlier, dynamic pressure changes have been observed to cause vortical flow patterns in the ionosphere. Hence it is important to assess such effects as a possible cause of the events presented. The radar was observing equatorward of the persistent cleft aurora, and hence using the discussion given by Elphic [1988], Glassmeier et al. [1989] and Lee (submitted, 1990), we can define the flow sequences we would expect to observe. For a twin vortex pattern, Figure $1 d$ shows that we would expect to see the following sequence of flow directions at the radar: southeast then northeast then northwest then southwest for westward propagation of a pair of field-aligned currents led by the downward one. If the upward current leads, the same sequence, but for the opposite flow senses, would be observed. For eastward propagating events the reverse sequences would be observed. For each of a series of single vortices, the sequence would be north then west then south for an eastward moving upward field-aligned current filament: the same sequence but of reversed flows would be observed for a downward current filament, and again the sequences would be reversed for eastward propagation. None of these sequences match the repetitive west then north flows seen by the optical instruments and by EISCAT on January 12, 1988. The plasma flow speeds are derived from the radar beam-swinging technique. However, the flow magnitudes are undoubtedly as large as quoted $\left(2-3 \mathrm{~km} \mathrm{~s}^{-1}\right)$ : Lockwood et al. [1989c] show that these flow 
bursts drive highly non-Maxwellian plasma ( $D^{*}$ values up to the toroidal threshold of 1.2 , even if the ion gas is assumed to remain pure $\mathrm{O}^{+}$; in general the large drifts will generate molecular ions, which means that this represents an underestimate in $\mathrm{D}^{*}$ ) and non-Maxwellian analysis shows that $\mathrm{T}_{i}$ exceeds $3000 \mathrm{~K}$ within flow bursts, but is $1000 \mathrm{~K}$ when the drift is low. From equation (2), this requires drifts in excess of 2 $\mathrm{km} \mathrm{s} \mathrm{s}^{-1}$, even if the neutral wind, $U$, is zero. In practice, $U$ would be expected to exceed $500 \mathrm{~m} \mathrm{~s}^{-1}$ westward after such a prolonged period of strong, westward ion flow, and $V$ must be nearer $3 \mathrm{~km} \mathrm{~s}^{-1}$.

In principle, plasma flow speeds of this magnitude could be generated by dynamic pressure pulses. The speed of these flows depends upon the height-integrated ionospheric Pedersen conductivity, $\Sigma_{p}$, and the magnitude of the field-aligned ${ }^{2}$ currents: the latter in turn depends upon the spatial gradients near the magnetopause and the magnitude of the pressure pulse. Hence if pressure pulse, spatial gradient and conductivity were all large enough, large ionospheric flow speeds could be generated. Heikkila et al. [1989] have recently reported $1 \mathrm{~km} \mathrm{~s} \mathrm{~s}^{-1}$ flows which they associate with an inferred pressure pulse. Todd et al. [ 1986] have observed $1.5 \mathrm{~km} \mathrm{~s}^{-1}$ flows directly in an event which Sibeck et al. [1989a] associate with a $0.5-\mathrm{nPa}$ pressure pulse. As pointed out by Lanzerotti [1989], the presence of a pressure pulse does not eliminate other possible causes of these events. From the predictions of Iee (submitted, 1990) we find that flows of $3 \mathrm{~km} \mathrm{~s}^{-1}$ could be generated by large pressure pulses ( $>2 \mathrm{nPa}$ ) if $\Sigma_{\mathrm{P}}$ is low ( $<1$ mho). We note that Sandholt et al. [1990] inferred a larger Pedersen conductivity than this during event 2 ( 6.6 mhos), but this did fall to 1.7 mhos outside the 557.7-nm transient aurora.

However, a very important finding of this paper is that the plasma motion is comparable with the event motion for every event. This has been shown to be true to a high degree of agreement for events 1 and 2 by comparing radar flow data and the auroral motions (here and by Sandholt et al. [1990]) and for all events, the northward speed of the westward flow channels, $V_{1}$, has been found to be similar to the northward plasma speed within the flow channels, $V_{n p}$. More precisely, $\left\langle\mathrm{V}_{n \mathrm{p}}\right\rangle /\left\langle\mathrm{V}_{\mathrm{p}}\right\rangle$ another problem for a dynamic pressure pulse interpretation because the speed of the event motion depends upon the fast mode velocity at the magnetopause and on the mapping of the field lines into the ionosphere: on the other hand, the speed of plasma motion depends upon the size of the pressure pulse, the spatial gradients at the magnetopause and the Pedersen conductivity. That the two are so similar that their ratio is between 1 and 1.5 is a highly unlikely coincidence, but one that was repeated for all nine events described here, despite the probable variation in magnitude of any dynamic pressure pulses.

Perhaps the greatest problem for any interpretation of the flow pattern in terms of pressure pulse effects is that flow direction in the event center, as defined by the radar, is the same as that of the event as a whole (as defined by the optical observations). In the introduction we discussed how this would not be expected for a dynamic pressure change twin vortex, where flow at the event center is expected to be at a large angle to the direction of event motion. In addition, the flows should be clearly vortical whereas the data presented here show strong fllow channel with only weak vortical return flows. In conclusion, al though Elphic [1988), Southwood and Kivelson [1990] and Iee (submitted, 1990) have together provided a plausible theory by which dynamic pressure changes can produce vortical flows within the dayside auroral ionosphere, the characteristics of the observations discussed here are not consistent with this theory.

During the observations on January 12, 1988, direct solar wind data were not available. However, the IMF data have been presented by Sandholt et al. [1989a]. The IMF field strength, $B$, is shown here in Figure 11 (15-s averages of raw data). In general, the field magnitude is relatively constant, but there are a number of small depressions. The arrows marked a- $u$ in Figure 11 show all events where $B$ decreases by more than $1 \mathrm{nT}$ in $1 \mathrm{~min}$ and remains at the depressed value for at least $30 \mathrm{~s}$. As mentioned earlier, it is of ten suggested that such decreases in $B$ correspond to increases in thermal pressure such that the total pressure (sum of thermal and magnetic) is constant, as has been observed by Burlaga [1968]. Furthermore, if the temperatures are assumed constant, then this must be due to an increase in solar wind density, and hence depressions in B may denote an increase in solar wind dynamic pressure. All depressions in Figure 11 are less than $3 \mathrm{nT}$. However, using solar wind electron and ion temperatures of $10^{5} \mathrm{~K}$ and $2.4 \times 10^{4}$ $\mathrm{K}$, respectively, as derived by Burlaga, and the estimated solar wind speed of $500 \mathrm{~km} \mathrm{~s}^{-1}$ at this time [see Lockwood et al., 1989b] the $\Delta \mathrm{B}$ of $1 \mathrm{nT}$ to $3 \mathrm{nT}$ correspond to dynamic pressure increases of between $1.1 \mathrm{nPa}$ and $6.6 \mathrm{nPa}$. These are large pressure pulses, even compared with the mean value of $5 \mathrm{nPa}$ derived using the mean solar wind density estimate of $10^{7} \mathrm{~m}^{-3}$ for this period, as given by Lockwood et al. [1989b]. However, caution is required as assumptions in their derivation may cause these dynamic pressure changes to be

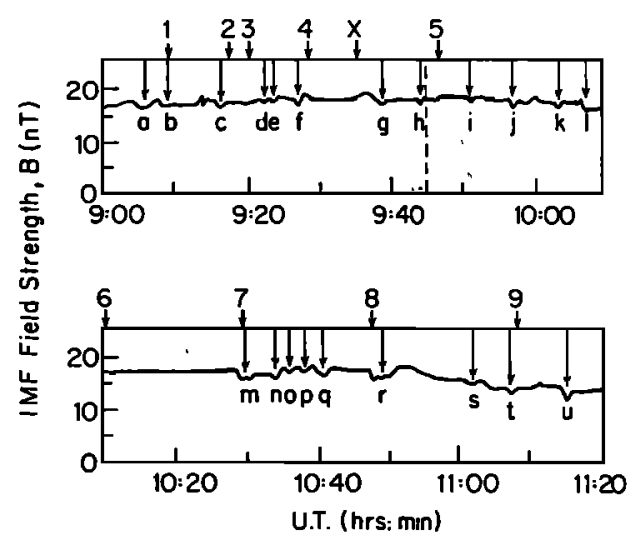

Fig. 11. IMF field strength, B, observed on January 12, 1988. Events marked a-u are depressions in $\mathrm{B}$ exceeding $1 \mathrm{nT}$ within $1 \mathrm{~min}$ and lasting a further $30 \mathrm{~s}$. The arrows marked $1-9$ and $X$ give the times of peak voltage, $\Phi$, in the radar/auroral events, lagged by the satellite to radar delay, $\mathrm{T}_{\mathrm{Sr}}$, computed by Lockwood et al. [1989b]. 
overestimates (as for the January 15 event discussed earlier). The occurrence of these $B$ decreases (and hence inferred solar wind dynamic pressure pulses) does not, however, match that of the auroral/flow burst events seen in the ionosphere. The times of peak vol tage, $\Phi$, of the events observed from the ground are given in Figure 11 by the arrows marked $1-9$ (and including the extra event $X$ discussed by Lockwood et al. [1989b]): these times have been advanced by the predicted satellite-to-radar propagation delay, $\mathrm{T}_{\mathrm{Sr}}$, computed by Lockwood et al. [1989b] . Thi's delay assumes that the pressure pulse is aligned with the IMF vector in the $\mathrm{X}-\mathrm{Y}$ plane; however, our conclusions do not depend critically upon this assumption as results for assuming the pressure pulse is aligned perpendicular to the solar wind flow, for example, produce the same general behavior. Some of the IMF decrease events (a-u) do line up well with the events observed from the ground (for the delay $T_{\text {rr }}$ these are $1,2,4,6,7,8$ and 9 ), but other ${ }^{5 r}$ ionospheric events (e.g. $3, X$ and 5 ) have no clear associated IMF decrease and many IMF decreases ( $a, d, e, g$, $h, i, j, k, l, n, o, p, q, s$ and $u$ ) appear to cause no ionospheric event, even though some of them are of the larger ( $3 \mathrm{nT}$ ) armi itude (e.g. $\mathbf{a}, \mathbf{g}, \mathbf{n}, \mathrm{q}$ and $\mathrm{u}$ ). The dashed line is at $0945 \mathrm{UT}$ when the IMF ceased to be continuously southward. Prior to this time Lockwood et al. [1989b] show that the auroral/flow burst events repeat every $8.3 \mathrm{~min}$. Here we find the equivalent recurrence period of the decreases in $B$ is $5.6 \mathrm{~min}$. In the interval 0945-1110, the ionospheric events recur every $19.2 \mathrm{~min}$, on average, whereas the corresponding figure for the IMF decreases is 6.5 min. Hence there is no significant drop in the rate of IMF decreases when the IMF turned predominantly northward at 0945 UT (as one would expect), whereas the ionospheric $\in$ vents are less frequent by a factor of about 2.5. Lockwood et al . [1989b] have shown that after 0945, each

ionospheric everit could have been triggered by an isolated swing to southward IMF and each southward swing could be associated with an ionospheric event. We do not think the IMF decreases are conclusive evidence for pressure pulses, but there is certainly no evidence that they were associated with the auroral/flow burst events. Furthermore, the IMF had a strong positive $B_{y}$ component at this time and hence there is no ${ }^{\mathrm{y}}$ reason to expect pressure oscillations in the magnetosheath (but which are absent from the solar wind) - such effects have been suggested for radial IMF orientations [Fairfield et al., 1989].

Interpretation in Terms of Flux Transfer Events

The other model is that of transient reconnection. This was originally proposed to explain "FIE" observation at the magnetopause, and we have no direct evidence that links the magnetopause signatures with the transient dayside ionospheric phenomena discussed here. However, there are a great many features which do accord very well with the magnetopause observations. Firstly, the occurrence and repetition rate of the ground signatures is (from these limited available data) very similar to that for FIEs [Rijnbeek et al., 1984; Berchem and Russell, 1984]. The motion of the events (west then north) is exactly as predicted for the effects of magnetic tension and magnetosheath flow on newly opened flux tubes, and the release of magnetic tension can give the large $\left(2-3 \mathrm{~km} \mathrm{~s}^{-1}\right)$ westward flow speeds observed [Lockwood and Freeman, 1989; Saunders, 1989]. The flow at the event center is found to be the same as the event motion (in both speed and direction): an important condition for any FTE signature. This finding is subject to some uncertainty however, primarily due to the effects of the radar beamswinging technique employed and the requirement to assume an emission altitude when comparing with optical aurorae. More accurate determination of plasma flow vectors during these events is an important scientific aim of the proposed radar on Svalbard, to work in conjunction with EISCAT [Cowley et al., 1990]. In addition, the planned use of multiple sites on Svalbard for optical observations would remove the requirement to assume the emission altitude. Until such experiments are carried out, we can only say that the event motion and plasma motion are comparable. However, we believe that the similarity is close enough to strongly suggest the events are FTEs (and strongly suggest that they are not dynamic pressure pulse effects).

There is good evidence that most of the events are elongated in the east-west direction. This of fers an explanation of the weakness of the eastward flow regions outside the events, as shown in Figures $5 \mathrm{a}$ and 10. It should here be noted that Figure 10 only shows the flows due to an isclated FIE, and no other sources of flow are considered. In general the flows excited by the FTE will be superposed on background flows due to other sources. In particular, Lockwood et al. [1990] point out that al though dayside flows are dominated by reconnection at the dayside magnetopause, reconnection in the geomagnetic tail also drives some flow on the dayside. We would therefore expect some westward flow on closed field lines in the afternoon sector due to reconnection in the tail, and the FTE flow pattern will be superposed on this. Because the "return" flows outside the region of newly opened flux are so weak when the event is elongated and moving along its long axis (as show in Figure 10), the net flow on closed field lines equatorward of the event may well still be westward (but at much lower speeds than the westward flow within the region of newly opened flux and also at lower speeds than the flow on closed field lines before and after the event), for example as seen in the event 2 [Sandholt et al., 1990, figure 2]. The elongation is also in good agreement with the all-sky TV images [Sandholt et al., 1989a]. Theoretically this strongly supports the Southwood et al. [1988]/Scholer [1988] model of FTEs, which invokes time-dependent reconnection at a single, elongated neutral line. We identify the region of transiently enhanced 630-nm emission with the newly opened flux caused by the burst of enhanced reconnection. The 557.7-nm arc covers a smaller region which appears to coincide with the upward field-aligned current region in the Southwood FTE model. From these considerations we find that the open flux tube has dimensions of $300 \mathrm{~km}$ (northsouth) by about 1500 to $2000 \mathrm{~km}$ (east-west) in the ionosphere. From simplistic field line mapping, we esimate that magnetopause signatures may be $2 R_{F}$ in the dimension aligned with their motion but $10-15 R_{E}$ in the dimension 
perpendicular to their motion; the latter being the length of the reconnection neutral line on the magnetopause. As discussed in the introduction, this mapping is very approximate and assumes the newly opened flux is appended to a pre-existing polar cap [Crooker, 1990, Crooker and Siscoe, 1990].

Table 1 shows that the lifetimes, T, of the flow channels vary between $7.5 \mathrm{~min}$. (event 4) and $27.5 \mathrm{~min}$ (event 5) and values are generally larger than the $\sim 10 \mathrm{~min}$ for which Lockwood et al. [1990] predict that newly opened flux should excite convection. This latter result was obtained from EISCAT-AMPTE observations of the response of dayside convection to changes in the IMF and previous analyses of the response of geomagnetic disturbance. Furthermore, in most cases the lifetime of the flow channel is limited because it moves poleward of the radar field of view, not because it fades within it. Hence values of $T$ would appear to be minima. Exceptions appear to be events 5 and 8 , for which $V_{\text {TT }}$ falls to near the adopted $1 \mathrm{~km} \mathrm{~s}^{-1}$ event ${ }^{\text {Wp }}$ threshold toward the end of their lifetime. For these two cases, T is large (27.5 min and $22.5 \mathrm{~min}$, respectively). We shall take 25 min to be the typical lifetime of the later, longer-lived events (i.e., events 5-9). It is interesting to note that these longer-lived events are those for which the IMF was predominantly northward but showed isolated swings to a southward orientation at the time required to trigger an event [Lockwood et al., 1989b].

Some of the difference between these values and the $\sim 10$ min derived by Lockwood et al. can be accounted for by the time taken to reconnect the newly opened flux. If reconnection commences at time $t=0$, the first open flux will excite ionospheric flow from $t=d$, where $d$ is the magnetopause-to-ionosphere propagation delay. If the last flux is reconnected at $t=\Delta t$, it will excite flows for the period $(\Delta t+d)$ to $(\Delta t+d+e)$, where $e$ is the time for which each flux tube excites convection. Hence $T=(\Delta t+d+e)-d=(\Delta t+e)$. The duration of satellite observations of FTEs is about 1-5 min, with values typically being $2 \mathrm{~min}$. If the newly reconnected flux moves over the magnetopause at constant speed, then this will be the time taken to reconnect the open flux, $\Delta t$. If however, the event is accelerated between the $\mathrm{X}$ line and the satellite (as the field lines unbend), $\Delta t$ will be larger than the duration of the magnetopause signature. We also note that for the Southwood et al./Scholer model, Scholer [ 1988, 1989] simulated bubbles of typical FTE scale size (i.e., 1 Farth radius in the direction along the magnetopause normal to the $X-1$ ine) for a burst of reconnection lasting $\Delta t=2 \mathrm{~min}$. Subtracting this $\Delta t$ from the given values for $T$ for each event yields e of about about $5-25 \mathrm{~min}$. The first two columns of Table 2 give the lifetimes and total magnetic flux of each event: an elliptical flux tube has been assumed so the flux, F, is given by the product of the area $(\pi a b / 4$, where $a$ and $b$ are the ellipse axes lengths given in Table 1) and $B_{i}$, the ionospheric magnetic field. The third column of Table 2 gives the voltage along the $X$ line, $V_{2}$, required to reconnect the tube in a time $\Delta t$ of ${ }^{2}$ $2 \mathrm{~min}$. These voltages are very large, particularly for the later events, for which $\mathrm{T}$ is also large. The reconnection vol tage may, in reality, not have been this great, and hence the time $\Delta t$ would be
TABLE 2. Lifetimes of Flow Channels, T, Total

Magnetic Fluxes of Inferred Open Flux Tubes $\left(F=B_{i} \pi a b / 4\right)$ and Required Reconnection

${ }^{1}$ Voltages for a Reconnection Time, $\Delta t$, of $2 \min .\left(V_{2}\right)$

\begin{tabular}{lccc}
\hline $\begin{array}{l}\text { Event } \\
\text { Number } \\
\text { (see }\end{array}$ & $\begin{array}{l}\text { Lifetime } \\
\text { of flow } \\
\text { channel, } \\
\text { Figure 2) }\end{array}$ & $\begin{array}{l}\text { Magnetic } \\
\text { Flux, F, } \\
10^{7} \text { Wb }\end{array}$ & $\begin{array}{l}\text { Voltage, } \\
\mathrm{V}_{2},\end{array}$ \\
\hline 2 & 750 & 1.9 & 158 \\
3 & 900 & 1.5 & 125 \\
4 & 450 & 0.7 & 58 \\
5 & 1650 & 1.8 & 150 \\
6 & 1350 & 2.4 & 200 \\
7 & 1350 & 2.6 & 217 \\
8 & 1350 & 3.4 & 283 \\
9 & 1500 & 2.9 & 242 \\
\hline
\end{tabular}

correspondingly larger. Specifically we would suggest from typical transpolar voltages that the reconnection voltage may have been under half the given $\mathrm{V}$ for the larger $\mathrm{T}$ events, giving

$\Delta t \approx 5^{2}$ min. This is less than the duration of the isolated swings of the IMF to southward which Lockwood et al. [1989b] associate with these later events (and would be the trigger in an FTE interpretation) but is at the top end of inferred reconnection times from magnetopause observations. Note also that the Scholer [1989] simulations would suggest a bubble diameter of about $2 \mathrm{R}_{\mathrm{F}}$ for this $\Delta t$, consistent with the values of a in Table 1. Using the typical $T$ for events 5 to 9 of 25 min, this $\Delta t$ yields $e \approx 20 \mathrm{~min}$.

In addition, the value for e quoted by Lockwood et al. [1990] was envisaged to relate to the time for the antisolar velocity of newly opened flux tubes to fall to the background level, i.e., for them to be wrapped over the front edge of the tail lobe and subsumed into the polar cap flow. We suggest that in the case presented here, because of the larger than average $B_{y}$ IMF component, the flux tubes spend longer than average moving westward around the polar cap boundary, which would largely occur before the antisolar motion begins. Two other points should be remembered. First, Iockwood et al. did not state that flux tubes excite no convection more than 10 min after convection, but that their effect was small after this time (indeed they note that Todd et al. [1988] observed a weak, but slowly-decaying residual effect after this time). Second, the lifetimes, $T$, given in Table 2 would be considerably reduced if a higher threshold for $V$ were adopted in the definition of a flow channel. In Table 2, $\mathrm{V}_{\mathrm{w}}>1 \mathrm{~km} \mathrm{~s}$ is used. However, Figure $2 c$ shows that ${ }^{W p_{\text {for }}}$ many events $\mathrm{V}_{\text {wp }}$ is near this limit for several minutes early ${ }^{\text {Pp }}$ in the event lifetime and increasing this threshold condition to just $1.2 \mathrm{~km} \mathrm{~s}^{-1}$ shortens events $5,6,7,8$ and 9 by $10,10,10,7.5$ and $2.5 \mathrm{~min}$, respectively.

Hence it seems that lifetimes of flow channeis may be slightly longer than would be predicted from the results of Lockwood et al. [1990], even allowing for the reconnection time of the newly opened flux. This may in part be due to the large IMF $Y$ component, allowing flux tubes to slip a long way around the front of the lobe, before moving into it and being subsumed into the polar 
cap flow. However, considering the uncertainties caused by defining the background flow level on which the events are superposed, we consider the observed lifetimes to be in good agreement with the conclusions of Lockwood et al.

Table 2 also gives an indication of the contribution to the mean transpolar voltage due to these events. During the period 0915:00-0937:00UT, we observed events 2,3 and 4 , and the IMF was continuously southward. (Note that this period has been chosen so that event 1 and the possible event $X$, as discussed by Lockwood et al. [1989b] were excluded because it was difficult to define the dimension $b$, and hence $\mathrm{F}$, for these events.) Adding the magnetic flux estimate, $F$, for these three events, we find that a total of $4.1 \times 10^{7} \mathrm{~Wb}$ was added to the polar cap during this interval (of duration $1350 \mathrm{~s}$ ), corresponding to a mean voltage $\langle\mathrm{V}\rangle=4.1 \times 10^{7} / 1350=30 \mathrm{kV}$. We note that the the mean value of IMF $B_{7}$ for this interval was $\approx-5 n$. Using the estimated average solar wind speed $\mathrm{v} \approx 450 \mathrm{~m} \mathrm{~s}^{-1}$ for this period [Lockwood et al. $S W_{1989 b}$ we estimate that the motional solar wind electric field, $E=\left(v_{S w} B_{z}\right)$, to have been $2.25 \mathrm{mV} \mathrm{m}^{-1}$, for which ${ }^{\mathrm{SW}}$ the $_{\mathrm{z}}$ scatter plot by Cowley [1984b] yields a mean transpolar voltage of roughly $100 \mathrm{kV}$ ( \pm about $30 \mathrm{kV}$ ). On this basis, we estimate that FIFs contribute of order $30 \%$ of the total transpolar voltage for strongly southward IMF.

As discussed by Wright [1987] and Southwood et al. [1988], a reconnected flux tube will be twisted and will subsequently untwist, giving vortical motion in the ionosphere [Lee, 1986]. For a circular flux tube, this merely generates vortical flows within the open tube (Figure 1). However, for the greatly elongated tubes inferred here the untwisting would also cause considerable flows outside the tube (like a rotating paddle). It is interesting to note from the auroral images that the events appear to remain aligned with the polar cap boundary. If they are FTE flux tubes, this implies that they untwist by the angular length of the boundary they move along. In Figure 10 we sketch this to be 4-5 hours of MLT, i.e., there appears to be an initial quarter twist on the tube which untwists as the event propagates west under magnetic tension.

\section{Discussion of Event on January 15, 1988}

The EISCAT data from January 15, 1988, provide an interesting comparison to those discussed above. The event here is observed to straddle the convection boundary reversal and move in the opposite direction at the same MLT. The flows and temperatures from all range gates are well explained by the FIE model, but for a flux tube which is nearly circular (if anything it is longer in the north-south dimension). The vortical flows of the plasma appear to be inconsistent with a dynamic pressure change effect, and no clear dynamic pressure pulse is observed in the upstream solar wind at the interval when any event trigger would be present: however, at this time the IMF was observed to twice swing strongly southward. The IMF is near radial at this time, and it has been suggested that the bow shock can under such conditions introduce 10-min period oscillations in magnetosheath dynamic pressure [Fairfield, 1990; Sibeck et al., 1989c]. However, the event represents a single isolated event and does not appear to be one of a string of oscillations.

Iastly, using the ion temperature enhancements we can define that the pattern as a whole (note this includes a region of westward plasma flow) is moving eastward at $1.1 \mathrm{~km} \mathrm{~s}^{-1}$, the same as the velocity at the inferred event center. Note that the observations show both an eastward flow region and a westward flow region south of it, and both are moving eastward with this speed. In the introduction, we discussed how this is a necessary condition for the Southwood FTE ionospheric model but is not expected for the dynamic pressure vortices.

\section{Conclusions}

We conclude that none of the events discussed in this paper are consistent with current theories of the effects of dynamic pressure changes at the magnetopause but all are well explained in terms of the ionospheric signature of FTEs. Hence although dynamic pressure changes do undoubtedly cause large traveling vortical flows in the dayside auroral ionospinere, which have in the past been attributed to FTEs, we stress that not all such effects can be attributed to dynamic pressure, at least using current theories. Using the FIE interpretation, we find that the newly opened flux tubes in the ionosphere are typically $250 \mathrm{~km}$ in north-south extent, but a remarkable $1500-2000 \mathrm{~km}$ in east-west extent. This is strongly suggestive of the Southwood et al./Scholer FTE model of time-dependent reconnection at an elongated netral line. Modeling of the effects of elongated newly opened flux regions in FTES suggests that the vortical nature of ionospheric FTE signatures may not be very pronounced, and that they appear as "flow channels" anc current electrojets throughout the dayside auroral ionosphere.

This initial evidence suggests that FTEs contribute roughly $30 \%$ of the transpolar vol tage when the IMF is strongly southward $\left(\mathrm{B}_{\mathrm{z}} \approx-5 \mathrm{nT}\right)$.

Acknowledgments. We are grateful to the Director and staff of EISCAT for their help. EISCAT is supported by the Research Councils of France (CNRS), West Germany (MPG), Norway (NAVF), Sweden (NFR), Finland (SA) and the UK (SERC). We are also grateful to A. D. Lazarus for provision of the IMP 8 solar wind data and to K. S. C. Freeman for processing the EISCAT data. We thank D. J. Southwood and D. Sibeck for helpful discussions.

The Editor thanks D. G. Sibeck and E. A. Bering for their assistance in evaluating this paper.

\section{References}

Berchem, J., and C. T. Russel1, Flux transfer events at the magnetopause: Spatial distribution and controlling factors, J. Geophys. Res., 89, 6689-6703, 1984.

Bering, E. A., J. R. Benbrook, G. J. Byrne, B. Liao, J. R. Theall, I. J. Ianzerotti, C. G. MacLennan, A. Wolfe, and G. I. Siscoe, Impulsive electric and magnetic field perturbations observed over South Pole: Flux transfer events?, Geophys. Res. Iett., 15, 1545-1548, 1988. 
Bering, E. A., L. J. Lanzerotti, J. R. Benbrook, Z. -M. Lin, C. G. Maclennan, A. Wolfe, R. E. Lopez, and E. Friis-Christensen, Solar wind properties observed during high-latitude impulsive perturbation events, Geophys. Res. Lett., 17, 579-586, 1990.

Bowe, G. A., M. A. Hapgood, M. Lockwood, and D. M. Willis, Short-term variability of solar wind number density, speed and dynamic pressure as a function of the interplanetary magnetic field components: A survey over two solar cycles, Geophys. Res. Iett., in press, 1990.

Burlaga, I. F., Micro-scale structures in the interplanetary medium, Sol. Phys., 4, 67-92, 1968.

Cowley, S. W. H., The causes of convection in the Earth's Magnetosphere: A review of developments during IMS, Rev. Geophys., 20, 531- 565, 1982.

Cowley, S. W. H., Evidence for the occurrence and importance of reconnection between the Farth's magnetic field and the interplanetary magnetic field, in Magnetic reconnection in space and laboratory plasmas, Ceophys. monogr. Ser., vol. 30, edited by E.W. Hones, Jr., pp. 375- 378, AGU, Washington, D. C., 1984a.

Cowley, S. W. H., Solar wind control of magnetospheric convection, in Achievemnts of the International Magnetospheric Study, IMS. Eur. Space Agency Spec. Publ., SP-217, 483-494, 1984b.

Cowley, S. W. H., The impact of recent observations on theoretical understanding of solar wind-magnetosphere interactions, J. Geomagn. Geoelectr., 38, 1223-1256, 1986.

Cowley, S. W. H. , A. P. van Eyken, E. C. Thomas, P. J. S. Williams and D. M. Willis, The scientific and technical case for a polar cap radar, J. Atmos. Terr. Phys., in press, 1990.

Crooker, N. U., Flux transfer event footprint patterns and implications for convection, $\mathrm{J}$. Geophys. Res., 95, 10,567-10,573, 1990.

Crooker, N. U. and G. L. Siscoe, on mapping flux transfer events to the ionosphere, $\mathrm{J}$. Geophys. Res., 95, 3795-3799, 1990.

Dessler, A. J., Iength of the magnetospheric tail, J. Geophys. Res., 69, 3913-3918, 1964.

Elphic, R. C., Multipoint observations of the magnetopause: Results from ISEE and AMPTE, Adv. in Space Res., 8 (9), 223-238, 1988.

Etemadi, A., S. W. H. Cowley, and M. Lockwood, The effect of rapid change in ionospheric flow on velocity vectors deduced from radar beamswinging experiments, J. Atmos. Terr. Phys., 51, 125-138, 1989.

Fairfield, D., W. Baumjohann, G. Paschmann, H. Liuhr, and D. Sibeck, Upstream pressure variations associated with the bow shock and their effects on the magnetosphere, J. Geophys. Res., 95, 3773-3786, 1990.

Farrugia, C. J., R. P. Rijnbeek, M. A. Saunders, D. J. Southwood, D. J. Rodgers, M. F. Smith, C. P. Chaloner, D. S. Hall, P. J. Christiansen, and L. J. C. Woolliscroft, A multi-instrument study of flux transfer event structure, J. Geophys. Res., 93, 14,465, 1988.

Farrugia, C. J., M. P. Freeman, S. W. H. Cowley, D. J. Southwood, M. Lockwood, and A. Etemadi, Pressure-driven magnetopause motions and attendant response on the ground, Planet. Space Sci., 37, 589-607, 1989.

Fri is-Christensen, E., M. A. McHenry, C. R. Clauer, and S. Vennerstrøm, Ionospheric traveling convection vortices observed near the polar cleft: A triggered response to sudden changes in the solar wind, Geophys. Res. Iett., 15, 253-256, 1988.

Glassmeier, K.-H., M. Hoenisch, and J. Untied, Ground-based and satellite observations of traveling magnetospheric convection twin vortices, J. Geophys. Res., 94, 2520-2528, 1989

Goertz, C. K., E. Neilsen, A. Korth, K.-H. Glassmeier, C. Haldoupis, P. Hoeg and D. Hayward, Observations of a possible ground signature of flux transfer events, $\mathrm{J}$. Geophys. Res., 90, 4069-4078, 1985.

Heikkila, W. J., T. S. Jørgensen, L. J. Ianzerotti, and C. J. Maclennan, A transient auroral event on the dayside, J. Geophys. Res., $94,15,277-15,290,1989$

Ianzerotti, L. J., Comment on "Solar wind dynamic pressure variations and transient magnetospheric signatures," Geophys. Res. Lett. , 16, 1197-1199, 1989.

Ianzerotti, L. J., R. D. Hunsucker, D. Rice, L.-C. Lee, A. Wol fe, C. G. Maclennan and L. V. Medford, Ionosphere and ground-based response to field-aligned currents near the magnetospheric cusp region, J. Geophys. Res., 92, T739-7743, 1987.

Iee, I. C., Magnetic flux transfer at the Earth's magnetopause, in Solar wind-magnetosphere coupling edited by Y. Kamide and J. A. Slavin, pp. 297-314, Terra Scientific, Tokyo, 1986.

Lee, L. C., and Z. F. Fu, A theory of magnetic flux transfer at the Earth's magnetopause, Geophys. Res. Lett., 12, 105-108, 1985.

Iockwood, M., and S. W. $\bar{H}$. Cowley, Observations at the magnetopause and in the auroral ionosphere of momentum transfer from the solar wind, Adv. Space Res., 8 (9), 281-299, 1988.

Lockwood, M., and M. P. Freeman, Recent ionospheric observations relating to solarwind-magnetosphere coupling, Philos. Trans. R. Soc. Iondon, Ser. A., 328, 93-105, 1989.

Lockwood, M., and M. F. Smith, Low-al ti tude signatures of the cusp and flux transfer events, Geophys. Res. Iett., 16, 879-882, 1989.

Lockwood, M., and M. F. Smith, Reply to Newell, Geophys. Res. Iett., 17 305-306, 1990.

Lockwood, M., and K. J. Winser, on the determination of ion temperature in the auroral F-region ionosphere, Planet. Space Sci., 36, 1295-1304, 1988.

Lockwood, M., A. P. van Eyken, B. J. I. Bromage, D. M. Willis, and S. W. H. Cowley, Eastward propagation of a plasma convection enhancemnet following a southward turning of the interplanetary magnetic field, Geophys. Res. Lett., 13, 72-75, 1986.

Lockwood, M., s. W. H. Cowley, H. Todd, D. M. Willis and C. R. Clauer, Ion flows and heating at a contracting polar cap boundary, Planet. Space Sci., 36, 1229-1253, 1988 .

Lockwood, M., M. F. Smith, C. J. Farrugia, and G. I. Siscoe, Ionospheric ion upwelling in the wake of flux transfer everts at the dayside magnetopause, J. Geophys. Res., 93, 5641-5654, $1988 \mathrm{~b}$. 
Lockwood, M., P. E. Sandholt, and S. W. H. Cowley, Dayside auroral activity and magnetic flux transfer from the solar wind, Geophys. Res. Iett., 16, 33-36, 1989a.

Iockwood, $\overline{\text { M. }}$, P. E. Sandholt, S. W. H. Cowley, and T. Oguti, Interplanetary magnetic field control of dayside auroral activity and the transfer of momentum across the dayside magnetopause, Planet. Space Sci., 37, 1347-1365, 1989b.

Lockwood, M., K. Suvanto, K. J. Winser, S. W. H. Cowley and D. M. Willis, Incoherent scatter radar observations of non-Maxwellian ion velocity distributions in the auroral F-region, Adv. in Space Res., 9 (5), 113-113, 1989c.

Lockwood, M., S. W. H. Cowley, and M. P. Freeman, The excitation of ionospheric convection, $\mathrm{J}$. Geophys. Res., 95, 7961-7972, 1990.

Lundin, R., on the magnetospheric boundary layer and solar wind energy transfer into the magnetosphere, Space Sci. Rev., 48, 263-320, 1988.

McHenry, M. A., and C. R. Clauer, Modeled ground magnetic signatures of flux transfer events, J. Geophys. Res., 92, 11,231-11,240, 1987.

McHenry, M. A., C. R. Clauer, E. Fri isChristensen, and J. D. Kelly, Observations of ionospheric convection vortices: signatures of momentum transfer, Adv. in Space Res., 8 (9), 315-320, 1988.

Milne-Thompson, L. M., Theoretical Hydrodynamics, MacMillan, New York, 1955.

Paschmann, G., G. Haerendel, I. Papamastorakis, N. Sckopke, S. J. Bame, J. T. Gosling, and C. T. Russell, Plasma and magnetic field characteristics of magnetic flux transfer events, J. Geophys. Res., 87, 2159-2168, 1982.

Potemra, T. A., I. J. Zanetti, K. Takahashi, R. E. Erlandson, H. Liihr, G. T. Marklund, L. P. Block, and A. Iazarus, Multi-satelitte and surface observations of transient ULF waves, $\mathrm{J}$. Geophys, Res., 94, 2543-2554, 1989.

Reiff, P. H., and Luhmann, J. G., Solar wind control of the polar cap voltage, in Solar Wind-Magnetosphere Coupling, ed. Y. Kamide and J. A. Slavin, pp. 453-476, Terra Scientific, Tokyo, 1986.

Reiff, P. H., T. W. Hill, and J. L. Burch, Solar wind plasma injection at the dayside magnetospheric cusp, J. Geophys. Res., 82, 479491, 1977.

Ri jnbeek, R. P., S. W. H. Cowley, D. J. Southwood and C. T. Russell, A survey of dayside flux transfer events observed by the ISEE 1 and 2 magnetometers, J. Ceophys. Res., 89, 786-800, 1984.

Russel1, C. T., ana R. C. Elphic, Initial ISEE magnetometer results: Magnetopause observations, Space Sci. Rev., 22, 681-715, 1978.

Russell, C. T., and R. C. Elphic, ISEE observations of flux transfer events at the dayside magnetopause, Geophys. Res. Lett., 6 , 33-36, 1979.

Sandholt, P. E., IMF control of the polar cusp and cleft auroras, Adv. in Space Res., 8, 21-34, 1988.

Sandholt, P. E., A. Fgeland, J. A. Holtet, B. Lybekk, K. Svenes, and S. Asheim, S., Iargeand small-scale dynamics of the polar cusp, J. Geophys. Res., 90, 4407-4414, 1985.
Sandholt, P. E., B. Lybekk, A. Egeland, R. Nakamura, and T. Oguti, Midday auroral breakup, J. Geomagn. Geoelectr., 41, 371-387, 1989 a.

Sandholt, P. E., B. Lubekk, A. Egeland, B. Jacobson, P. F. Bythrow, and D. A. Hardy, Electrodynamics of the polar cusp ionosphere- A case study, J. Geophys. Res., 94, 6713-6722, 1989b.

Sandholt, P. E., M. Lockwood, T. Oguti, S. W. H. Cowley, K. S. C. Freeman, B. Lybekk, A. Egeland, and D. M. Willis, Midday auroral breakup events and related energy and momentum transfer from the magnetosheath, J. Geophys. Res., 95, 1039-1060, 1990.

Saunders, M. A., The origin of cusp Birkeland currents, Geophys. Res. Lett., 16, 151-154, 1989.

Saunders, M. A., C. T. Russell, and N. Sckopke, A dual-satellite study of spatial properties of FTEs, in Magnetic Reconnection in Space and Laboratory Plasmas, , Geophys. monogr. 30, edited by E. W. Hones, Jr., pp. 145-152, AGU, Washington, D. C., 1984.

Scholer, M., Magnetic flux transfer at the magnetopause based on single $X$-line bursty reconnection, Geophys. Res. Lett., 15, 291-294, 1988.

Scholer, M., Asymmetric time-dependent and stationary magnetic reconnection at the dayside magnetopause, J. Geophys. Res., 94, 15,099, 1989.

Sibeck, D. G., W. Baumjohann, and R. E. Lopez, Solar wind dynamic variations and transient magnetospheric signatures, Geophys. Res. Iett., $16,13-16,1989 \mathrm{a}$.

Sibeck, D. G., Baumjohann, W. and Iopez, R. E., Reply, Geophys. Res. Iett., 16, 1200-1202, 1989b.

Sibeck, D. G., W. Baumjohann, R. C. Elphic, D. H. Fairfield, J. F. Fennell, W. B. Gaill, L. J. Ianzerotti, R. E. Lopez, H. Liuhr, A. T. Y. Lui, C. J. Maclennan, R. W. McEntire, T. A. Potemra, T. J. Rosenberg, and K. Takahashi, The magnetospheric response to 8-min period strong-amplitude upstream pressure variations, J. Geophys. Res., 94, 2505-2519, 1989c.

Southwood, D. J., Theoretical aspects of ionosphere-magnetosphere-solar wind coupling, Adv. Space Res., 5 (4), 7-14, 1985.

Southwood, D. J., The ionospheric signature of Flux Transfer Events, J. Geophys. Res., 92, 3207-3213, 1987.

Southwood, D. J., and M. Kivelson, Ionospheric travelling vortex generation by solar wind buffeting of the magnetosphere, J. Geophys. Res., in press, 1990.

Southwood, D. J., M. A. Saunders, M. W. Dunlop, W. A. C. Mier-Jedrzejowicz, and R. P. Ri jnbeek, A survey of flux transfer events recorded by UKS spacecraft magnetometer, Planet. Space Sci., 34, 1349-1359, 1986.

Southwood, D. J., C. J. Farrugia, and M. A. Saunders, What are flux transfer events?, Planet. Space Sci., 36, 503-508, 1988.

St.-Maurice, J.-P., and W. B. Hanson, Ion frictional heating at high latitudes and its possible use for an in situ determination of neutral thermospheric winds and temperatures, J. Geophys. Res., 87, 7580-7602, 1982 
St.-Maurice, J.-P., and P.W. Schunk, Ion velocity distributions in the high-latitude ionosphere, Rev. Geophys., 17, 99-134, 1979.

Suvanto, K., M. Iockwood, K. J. Winser, B. J. I. Bromage and A. D. Farmer, Analysis of incoherent scatter radar data from nonMaxwellian F-region plasma, J. Atmos. Terr. Phys., 51, 483-495, 1989.

Todd, H., B. J. I. Bramage, S. W. H. Cowley, M. Lockwood, A. P. van Eyken, and D. M. Willis, EISCAT observations of bursts of rapid flow in the high latitude dayside ionosphere, Geophys. Res. Iett., 13, 909-913, 1986.

Todd, H., S. W. H. Cowley, A. Etemadi, B. J. I. Bromage, M. Lockwood, D. M. Willis, and H. Lihr, Flow in the high latitude ionosphere: Measurements at $15 \mathrm{~s}$ resolution made using the EISCAT "Polar" experiment, J. Atmos. Terr. Phys., 50, 423-446, 1988.

van Eyken, A. P., H. Rishbeth, D. M. Willis, and S. W. H. Cowley, Initial EISCAT observations of plasma convection at invariant latitudes 70$77^{\circ}$, J. Atmos. Terr. Phys., 46, 635-641, 1984.

Wei, C. Q., and I. C. Lee, Ground magnetic signatures of moving elongated plasma clouds, J. Geophys. Res., 95, 2405-2418, 1990.

Willis, D. M., M. Lockwood, S. W. H. Cowley, H. Rishbeth, A. P. van Eyken, B. J. I. Bromage,
P. R. Smith, and S. R. Crothers, A survey of simultaneous observations of the high latitude ionosphere and interplanetary magnetic field with EISCAT and AMPTE-UKS, J. Atmos. Terr. Phys., 48, 987-1008, 1386.

Wright, A. W., The evolution of an isolated reconnected flux tube, Planet. Space Sci., 35, 813-819, 1987.

S. W. H. Cowley, Blackett Iaboratory, Imperial College, London, SW7 2BZ, Fngland, United Kingdom.

R. P. Iepping, Iaboratory for Extraterrestrial Physics, Goddard Space Flight Center, Greenbelt, MD 20771.

M. Iockwood, Rutherford Appleton Iaboratory, Chilton, Didcot, OX11 OQX, England, United Kingdom.

P. E. Sandholt, Department of Physics, University of Oslo, Box 1048, Blindern, 0316 Oslo 3, Norway.
(Received November 28, 1989; revised May 14, 1990; accepted May 22, 1990.) 